\title{
Inter-Subunit Dynamics Controls Tunnel Formation During the Oxygenation Process in Hemocyanin Hexamers
}

OPEN ACCESS

Edited by:

Massimiliano Bonomi,

Institut Pasteur, France

Reviewed by:

Sophie Sacquin-Mora,

UPR9080 Laboratoire de Biochimie

Théorique (LBT), France

Simone Orioli,

University of Copenhagen, Denmark

*Correspondence: Shozeb Haider

Shozeb.haider@ucl.ac.uk

${ }^{+}$ORCID:

Shozeb Haider

orcid.org/0000-0003-2650-2925

Specialty section:

This article was submitted to Biological Modeling and Simulation, a section of the journal Frontiers in Molecular Biosciences

Received: 16 May 2021 Accepted: 23 August 2021 Published: 15 September 2021

Citation:

Bux K, Shen X, Tariq M, Yin J, Moin ST, Bhowmik D and Haider S (2021) InterSubunit Dynamics Controls Tunnel Formation During the Oxygenation Process in Hemocyanin Hexamers.

Front. Mol. Biosci. 8:710623.

doi: 10.3389/fmolb.2021.710623

\author{
Khair Bux ${ }^{1}$, Xiayu Shen ${ }^{2}$, Muhammad Tariq ${ }^{1}$, Junqi Yin ${ }^{3}$, Syed Tarique Moin ${ }^{1}$, \\ Debsindhu Bhowmik ${ }^{4}$ and Shozeb Haider ${ }^{2 * t}$
}

${ }^{1}$ Third World Center for Science and Technology, H.E.J. Research Institute of Chemistry, International Center for Chemical and Biological Sciences, University of Karachi, Karachi, Pakistan, ${ }^{2}$ UCL School of Pharmacy, London, United Kingdom, ${ }^{3}$ Oak Ridge National Laboratory, Center for Computational Sciences, Oak Ridge, TN, United States, ${ }^{4}$ Oak Ridge National Laboratory, Computer Sciences and Engineering Division, Oak Ridge, TN, United States

Hemocyanin from horseshoe crab in its active form is a homo-hexameric protein. It exists in open and closed conformations when transitioning between deoxygenated and oxygenated states. Here, we present a detailed dynamic atomistic investigation of the oxygenated and deoxygenated states of the hexameric hemocyanin using explicit solvent molecular dynamics simulations. We focus on the variation in solvent cavities and the formation of tunnels in the two conformational states. By employing principal component analysis and CVAE-based deep learning, we are able to differentiate between the dynamics of the deoxy- and oxygenated states of hemocyanin. Finally, our results identify the deoxygenated open conformation, which adopts a stable, closed conformation after the oxygenation process.

Keywords: hemocyanin, molecular dynamics, deep learning, metalloprotein, copper

\section{INTRODUCTION}

Hemocyanins (HCNs) are large type-3 metalloproteins that transport oxygen in the blood of invertebrates like arthropods and molluscs (Van Holde and Miller, 1995; Terwilliger, 1998; Burmester, 2002; Pick et al., 2008; Rehm et al., 2012; Marxen et al., 2014; Pinnow et al., 2016). Their binuclear active site coordinates copper as metal and accommodates oxygen as a peroxide ion, due to which copper oxidizes from (I) to (II) oxidation state (Himmelwright et al., 1978, 1980; Magnus and Ton-That, 1991; Jaenicke et al., 2012). Besides oxygen transportation, they have also been found to play a major role in diverse physiological functions like homeostasis, transportation of hormones (Prosser and Brown, 1961), in the development of immunity via activation of phenoloxidases protein that plays a major role in the formation of their exoskeleton formation and melamine pigments, as well as wound recovery (Lei et al., 2008; Coates et al., 2011; Coates and Nairn, 2014; Zhong et al., 2016; Coates and Talbot, 2018). In addition, HCNs also possess microbicidal property by enhancing the production of reactive oxidative (ROs) species that can develop an immediate defense mechanism against microbes. Furthermore, HCNs have also been reported to have antibacterial and antifungal properties (Dolashka et al., 2016; Qin et al., 2018). The Horseshoe crab (Limulus polyphemus) blue blood is being increasingly used in biotechnology and biomedical applications (Gibson III and Hilly, 1992; Riggs et al., 2002; Del Campo et al., 2011; Arancibia et al., 2012; Coates and Nairn, 2014; Gesheva et al., 2014; Kumar et al., 2015; Zhong et al., 
A Oxy- $-\mathrm{HCN}_{\text {monomer }}$

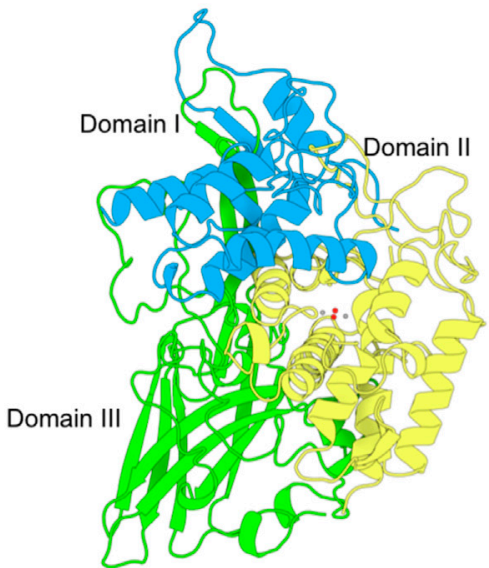

B Deoxy-HCN $\mathrm{H}_{\text {monomer }}$

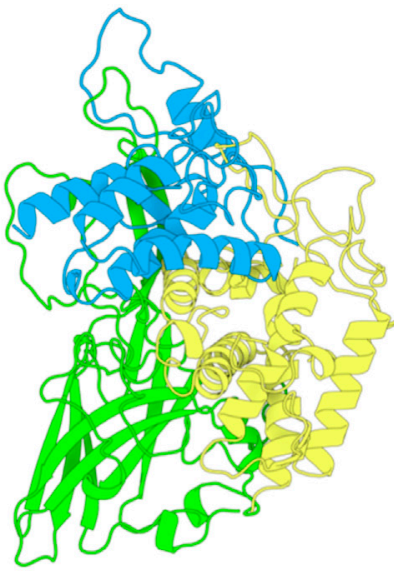

C

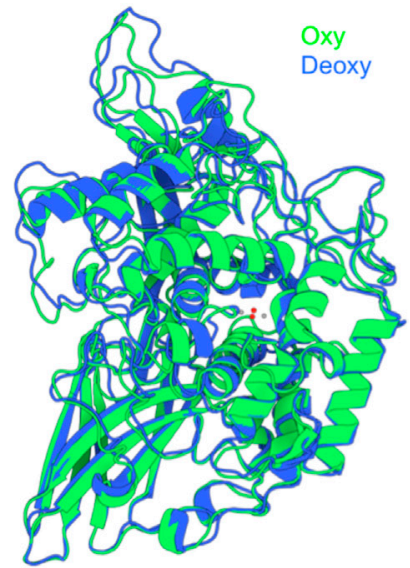

D Oxy- $\mathrm{HCN}_{\text {hexamer }}$
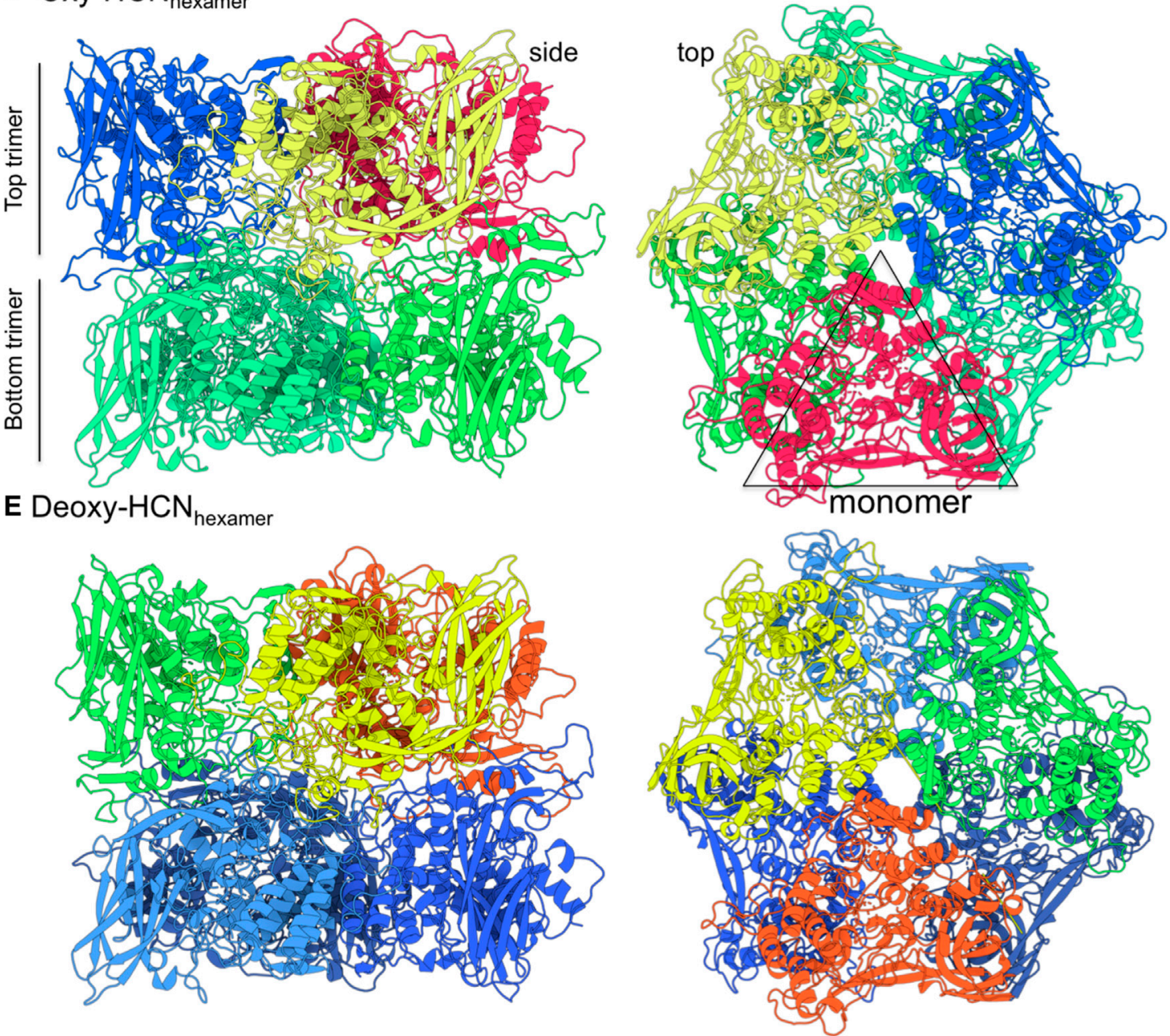

FIGURE 1 The structure of hemocyanin ( $\mathrm{HCN}$ ) from Limulus polyphemus in oxygenated (Oxy) and deoxygenated (Deoxy) states. (A,B) The structure is made up of six identical monomers. Each monomer consists of three domains. Domain II contains the copper binding site (grey spheres) that coordinates oxygen atoms (red spheres). (C) The monomers from Oxy and Deoxy states differ primarily in the conformation of the loops. (D,E) The structure is composed of two concentric layers of homotrimers and arranged as a "dimer-of-trimers." Monomer subunits in each top trimer are represented in a different color. The bottom trimer units are colored in green $(\mathrm{Oxy}-\mathrm{HCN})$ and blue (Deoxy-HCN). 
A

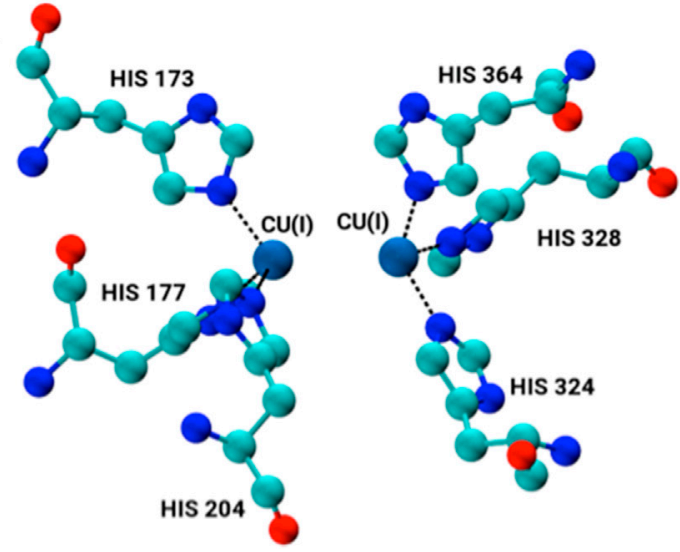

B

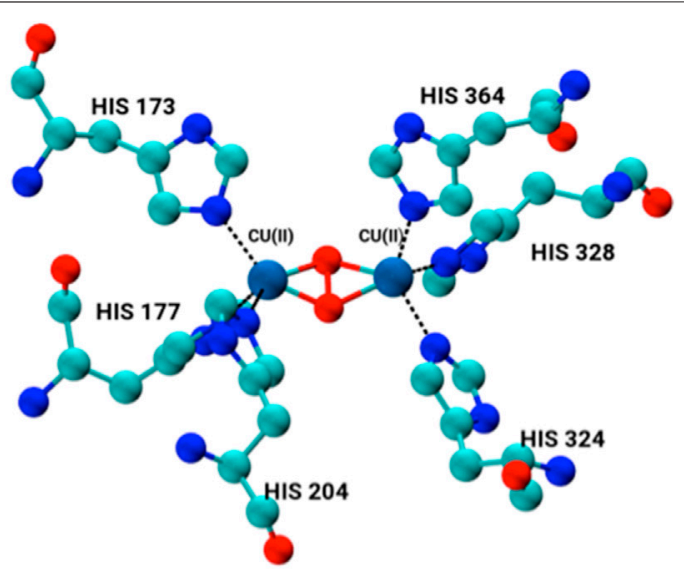

FIGURE 2 | Binuclear copper-containing metal binding site in (A) deoxygenated form and (B) oxygenated form of each monomer subunit within the hexamer.

2016; Besser et al., 2018). The copper rich blood contains amebocytes, which have an analogous role to the white blood cells in humans and are involved in the defense against pathogens. Thus, blue blood is used to produce Limulus amebocyte lysate (LAL), which has the potential to dysregulate the pathogenicity and virulence effects of pathogenic bacteria by reacting with their endotoxins that cause severe infections in the circulatory systems of humans. Moreover, HCNs have also been found to boost up the immune system of mammals by inducing the potent Th1-dominant immune response (Hanyecz et al., 2004). This property is being exploited in their use as immune stimulants and immune-modulators in cancer (Riggs et al., 2002; Matsushima et al., 2003; Arancibia et al., 2012; Becker et al., 2014; Gesheva et al., 2014; Zhong et al., 2016; Guncheva et al., 2019; Mora Román et al., 2019). More specifically, HCNs from Megathura crenulata (keyhole limpet hemocyanin) have shown anti-cancer properties in murine models of colon carcinoma, while being used as immuno-stimulants (Harris and Markl, 1999; Musselli et al., 2001; Miles et al., 2011). In addition, blue blood is widely used in the pharmaceutical industry to control the contamination of their products and medicines and in the development of antibodies and vaccines (Fellers and Harris, 1940; Levin and Bang, 1968; Levin et al., 1970; Berkson and Shuster Jr, 1999; Rutecki et al., 2004; Odell et al., 2005; Mazzotti et al., 2007; Bayer, 2016; Krisfalusi-Gannon et al., 2018; Maloney et al., 2018; Owings et al., 2019).

The X-ray structure of arthropod $\mathrm{HCN}$ is homo-hexameric (Figure 1). Two concentric rings, each consisting of three identical subunits, sit on top of another forming a dimer-oftrimers (Hazes et al., 1993; Magnus et al., 1994). Each subunit consists of three subdomains; 1) the C-terminal domain consists of residues 1 to $154 ; 2$ ) the $\mathrm{N}$-terminal domain contains residues from 380 to 628 ; and 3) the metal binding domain that lies between $\mathrm{N}$ - and $\mathrm{C}$-terminal domains contains residues ranging from 155 to 379 along with binuclear copper-containing active site (Volbeda and Hol, 1989; Hu et al., 2000; Hundahl et al., 2003; Matsushima et al., 2003). Each copper (I) atom forms a coordination bond with $\varepsilon$ nitrogen of three histidine residues, namely, His173, His177, His204 and His324, His328, His364. This represents the deoxygenated form of HCN (Deoxy-HCN). In the oxygenated form (Oxy-HCN) copper reversibly binds with oxygen resulting in the formation of the dicopper peroxo complex (Figure 2). There is subsequent oxidation of copper from $\mathrm{Cu}(\mathrm{I})$ to $\mathrm{Cu}(\mathrm{II})$ while retaining the coordination between three histidine residues and copper as observed in Deoxy-HCN (Decker and Tuczek, 2000; Hu et al., 2000).

The oxygenation mechanism of multimeric $\mathrm{HCN}$ is a complex phenomenon. Previous studies have reported on the detailed role of water in the oxygenation process, including its key role as an allosteric modulator of oxygen accessibility to the binuclear copper site of HCN (Hazes et al., 1993; Hundahl et al., 2003). Some studies have also reported that water in close proximity to copper (II) can also play an intimate role by replacing dioxygen molecule at the active site of HCN (Karplus and McCammon, 2002; Naresh et al., 2015; Bux et al., 2018). Furthermore, the formation of large solvent cavities at the interface of subunits has also been reported. It is via these cavities that the movement of oxygen up to the active site is possible (Magnus et al., 1994). The oxygenation process is also allosterically regulated such that the HCN structure acquires different conformational states (higher and lower oxygen affinity states) to attract oxygen to the active site. The allosteric changes are further dependent upon the dynamic behavior of protein that is entirely orchestrated by inter-residual dynamics of the subunits (Volbeda and Hol, 1989; Hazes et al., 1993; Magnus et al., 1994). HCNs have a negatively charged electrostatic surface, which makes solubility and subsequent hydration of HCNs in water possible (Coates and Nairn, 2013). A consequence of the hydration phenomenon is that it prevents the association of HCNs with other negatively charged entities that may affect the physiological function of the protein (Coates and Nairn, 2013).

The biological function of large biomolecules like $\mathrm{HCN}$ is dependent upon its conformational arrangement and cannot be explained solely on its structural description. Investigating the dynamic behavior of large biomolecules is, therefore, a key factor to understanding function. Experimental methods like X-ray 
crystallography are of great value in structural elucidation, but they are quite restricted in exploring the dynamical behavior because of its limitation in time and space resolution (Ohtaki and Radnai, 1993; Ma, 2005; Neutze, 2014). Theoretical and computational methods like molecular dynamics simulations (MD), on other hand, have emerged as the most reliable method to evaluate both structural and dynamical features of biomolecules (Sagui and Darden, 1999; Karplus and McCammon, 2002). In addition, conformational changes that mediate the oxygenation of $\mathrm{HCN}$ are entirely a dynamics dependent phenomenon. Therefore, a method that could provide microscopic insights into the dynamic behavior of the HCNs and variations as a function of time needs to be employed.

Many experimental studies have been reported for HCNs (Decker et al., 1988; Volbeda and Hol, 1989; Magnus and Ton-That, 1991; Hazes et al., 1993; Magnus et al., 1994; Jaenicke et al., 2012; Saito and Thiel, 2014; Naresh et al., 2015, 2015; Bux et al., 2018). Most of the up-to-date work commonly discusses the structural features of protein in its monomeric form and rarely in its biologically active hexameric form (Volbeda and Hol, 1989). Very few theoretical and computational studies have been reported for HCNs. Recently, in a combined QM/MM approach, the reversible binding of dioxygen at the binuclear copper active site was reported (Fariselli et al., 1999; Saito and Thiel, 2014). The study mainly focused on the active site of the protein and provides little insight into the structural dynamics of the multimeric protein. A previous study used MD simulations to report insights into the oxygenation mechanism of $\mathrm{HCNs}$ and the role of solvent via tunnel formation with the help of neighboring amino acid residues of the metal site (Bux et al., 2018). Again, the study was conducted using just one single subunit of $\mathrm{HCN}$ instead of the biologically active hexameric form.

Here, we present a detailed atomistic investigation of the hexameric HCN using MD simulations, with a focus on the conformations that the HCN adopts in oxygenated and deoxygenated states. By following the variation in solvent cavities and tunnels, we are able to identify residues that are involved in tunnel formation in Deoxy- and Oxy-HCN. Finally, our principal component analysis and CVAE-based deep learning results are able to differentiate between the global dynamics of these complex systems.

\section{METHODOLOGY}

\section{Modeling of the Hexamer}

The biologically active state of Limulus polyphemus (Atlantic horseshoe crab) HCN is homo-hexameric. Oxygenated (PDB id 1OXY; Hazes et al., 1993) and deoxygenated (PDB id 1LLA; Magnus et al., 1994) monomeric forms of HCN are available, while the hexameric form is yet to be reported. There are several missing loop residues in the monomeric form. Therefore, we first built the complete monomeric form using the Panulirus interruptus (California spiny lobster) structure as a template (PDB id 1HC1; Volbeda and Hol, 1989), which displays 62\% sequence homology. The modeled monomeric HCNs were then used to build a hexameric structure by structurally superimposing each subunit from horseshoe crab on hexameric spiny lobster structure. The loops were modeled using Modeller 9v23 (Webb and Sali, 2016) implemented in the Chimera suite (Pettersen et al., 2004).

\section{Active Site Modeling}

The active site of HCN is composed of two binuclear copper atoms each of which is coordinated to three histidine amino acid residues His172, His177, His204 and His324, His328, His 364. As a result of the bond between copper atoms and $\varepsilon$ nitrogen NE of the three histidine residues, the geometry of the copper coordination site becomes tetrahedral. The copper adopts oxidation state one $\mathrm{Cu}(\mathrm{I})$ in the deoxygenated form of the protein (Magnus and Ton-That, 1991; Hazes et al., 1993; Magnus et al., 1994; Fariselli et al., 1999). In the oxygenated form, the geometry of the binding site changes from tetrahedral to trigonal pyramidal. The oxidation state of copper changes from $\mathrm{Cu}$ (I) to $\mathrm{Cu}$ (II) upon binding of oxygen as a peroxide. In the present study, the active site in each subunit was modeled by constructing a bond between two copper atoms and the $\varepsilon$-NE atom in the imidazole rings of each six histidine residues, three on each side, in its both deoxygenated and oxygenated form (Figure 2).

\section{Force Field Parameterization of Copper Cluster}

The force field parameters for the copper-containing binuclear active site of the hexamer were constructed for the AMBER ff14SB force field using the bonded approach (Wang et al., 2004; Hornak et al., 2006). The bonded parameters for tetrahedral geometry for $\mathrm{Cu}(\mathrm{I})$ in deoxygenated and trigonal bi-pyramidal coordination for $\mathrm{Cu}(\mathrm{II})$ in an oxygenated state were taken from our previously reported study (Bux et al., 2018). Since HCNs are homo-hexameric, all six subunits contain the same residues in both deoxygenated and oxygenated states.

\section{Molecular Dynamics Simulations}

The ionization states of the amino acid side chains were determined at pH7.0, using propKa implemented in the play molecule (Martínez-Rosell et al., 2017). The system was described using the ff14SB force field and further processed to run MD simulations with Desmond 3.6 (Bowers et al., 2006). The overall charge was neutralized by manual addition of $150 \mathrm{Na}+$ ions to both systems that were subsequently solvated by adding TIP3P water molecules. In total, 77,896 (Deoxy) and 77,848 (Oxy) water molecules were present in a solvation box with edges set at least $12 \AA$ from the solute atom.

Equilibration of the hydrated systems was then carried out in an NPT ensemble for $50 \mathrm{~ns}$. Using the last frame from the equilibration, the production step was run in the NVT ensemble for both Oxy- and Deoxy-HCN systems. The SHAKE algorithm was used to simulate all bonds of hydrogen atoms in rigid and constrained form (Kräutler et al., 2001). Short bonded and nonbonded interactions were treated using RESPA integrator at the average time interval of about $2 \mathrm{fs}$ (Tuckerman 
et al., 1992) but longer time step of about 4 fs to simulate the long range interactions using Particle Mesh Ewald algorithms (Cerutti et al., 2009). The production step was run as 10 replicates of 100 ns each for both systems.

\section{Structural Analysis}

The trajectories were visualized using VMD (Humphrey et al., 1996). All analysis was carried out using GROMACS tools (Abraham et al., 2015) and pytraj (Roe and Cheatham, 2013) on the last $40 \mathrm{~ns}$ of each replicate, which was considered equilibrated after conventional RMSD analysis. The RMSD analysis was carried out using MDLovofit (Martínez, 2015). The radius of gyration $(\mathrm{Rg})$ was used to assess the compactness of the hexameric structure. The structural figures were generated in VMD (Humphrey et al., 1996) and Protein Imager (Tomasello et al., 2020).

\section{Dynamics Cross-Correlation Matrixes (DCCM) Analysis}

The dynamic behavior of hexameric $\mathrm{HCN}$ is a function of the correlated motions of individual subunits. The dynamic crosscorrelation matrices (DCCM) analysis investigates the correlated motions of amino acids that influence the dynamics of a protein (Kasahara et al., 2014). Bio3D suite (Grant et al., 2006) was used for DCCM analysis through which correlation motion was estimated by the projection of covariance matrix $\sigma$ between $2 \mathrm{C}_{\alpha}$ atoms $\mathrm{i}$ and $\mathrm{j}$ of proteins applying the following equation:

$$
\sigma_{i j}=\frac{\left\langle\Delta r_{i}(t) \cdot \Delta r_{j}(t)\right\rangle}{\sqrt{\left\langle\left\|\Delta r_{i}(t)\right\|^{2}\right\rangle \sqrt{\left\langle\left\|\Delta r_{j}(t)\right\|^{2}\right\rangle}}} .
$$

In the above equation, $r_{i}(t)$ defines the projected vector of atom $i$ as a function of time, whereas ensemble over average time is represented by $\langle "\rangle$ and change in position of $C_{\alpha}$ atoms, $i$ and $j$ with respect to their original position at a given time that is symbolized as $\Delta \mathrm{r}_{\mathrm{i}}(\mathrm{t})$ and $\Delta \mathrm{r}_{\mathrm{j}}(\mathrm{t})$. Correlation movements that were projected via estimation of matrices were then subsequently visualized through two-dimensional cross-correlation maps. The variation in correlative motion on these dynamics crosscorrelation maps was interpreted in terms of correlation (positive) and anti-correlation (negative) movements with respect to color appeared over maps. The last $40 \mathrm{~ns}$ from each trajectory were used for DCCM analysis.

\section{Principal Component Analysis (PCA)}

PCA is able to identify dominant motions and maximize variation during protein flexibility (Balsera et al., 1996). The results are presented as variations in the values of a small number of collective coordinates. The $\mathrm{Ca}$ coordinates were used as an input for PCA. More specifically, PCA was carried out to provide a quantitative and comparative analysis between the two states of the HCN hexamers. The dimensionality reduction was carried out using the PyPcazip program (Shkurti et al., 2016) and the porcupine plots were generated using in-house scripts (Haider et al., 2008). All the trajectories were combined so that all share the same subspace and comparisons can be made. A dot product matrix between the eigenvectors identified by the PCA on the Deoxy- and Oxy-HCN is calculated.

\section{Solvent Accessible Surface Area}

Solvent accessible surface area (SASA) can be used as a tool to assess the relative changes in conformational dynamics of homoand heterodimers or multimer unit proteins (Marsh and Teichmann, 2011). The experimental studies have reported that HCN can exist in two different states; open, before oxygenation, and closed after oxygen passes to the binding site. These acquired states are entirely dependent on the conformational integrity of the hexamer. Thus, an estimation of SASA allows us to observe changes in the conformation of individual subunits and the hexamer as a whole. SASA was calculated using the "gmx sasa" module as implemented in the GROMACS tools (Abraham et al., 2015).

\section{Interface Accessible Surface Area (IASA)}

The interactions at the interface of the top and bottom trimers in the dimer-of-trimers were evaluated by means of estimation of interface size or interface area through calculating the subsequent buried accessible surface area (ASA) upon complex formation among interface amino acid residues of individual subunits (Chen et al., 2013). Similarly, interactions at the interface of two dimers of trimers in hexamer were assumed to affect the entire interfacial dynamical properties of the whole protein.

The effect of contacts on the inter-subunit dynamics and the associated dynamical behavior of the hexamer was assessed by calculating the accessible surface area that is buried as a result of inter-subunit contacts between dimer-of-trimer in both Deoxyand Oxy-HCN via NACCESS (Hubbard and Thornton, 1993). Estimation of the interface accessible surface area (IASA) was carried in such a way that each trimer of the hexamer was assigned as two chains, $A$ and $B$ for which accessible surface area or change in the accessible area $(\triangle \mathrm{ASA})$ after complex formation was separately computed for chain $\mathrm{A}\left(\mathrm{ASA}_{\mathrm{A}}\right)$ and $\mathrm{B}$ $\left(\mathrm{ASA}_{\mathrm{B}}\right)$ with subsequently combined computation for both chains $\mathrm{AB}\left(\mathrm{ASA}_{\mathrm{AB}}\right)$ as explained in the following equation:

$$
\mathrm{IASA}=\mathrm{ASA}_{\mathrm{ChainA}}+\mathrm{ASA}_{\mathrm{ChainB}}-\mathrm{ASA}_{\mathrm{AB}} \text {. }
$$

The IASA was calculated over from 100 snapshots that were extracted from the last $40 \mathrm{~ns}$ from each replicate simulation.

\section{Solvent Cavity-Pocket Analysis}

Water is hypothesized to play a major role in passing oxygen up to the bi-nuclear copper-containing active site via cavities or tunnels formed within or at the interface of subunits. Evaluation of solvent cavities was thus carried out for both Oxy and Deoxy-HCN forms of the hexamer. Pocket analysis was done by calculating their opening and closing frequency in each individual subunit and then subsequently for the complete hexamer. Next, the cavities with maximum frequency were sorted and their volumes calculated. 200 snapshots from the last 40 ns of each replicate were used for pocket detection. MDPOCKET suite was used for this analysis (Schmidtke et al., 2011). 


\section{Tunnel Analysis}

The Caver 3.03 beta software (Chovancova et al., 2012) was used for the effective analysis of tunnels in structures derived from the molecular dynamics trajectories. This version uses an additional Voronoi diagram and is useful for the analysis of large macromolecules. For caver analysis, 330 frames were extracted from the last $40 \mathrm{~ns}$ of the Oxy- and Deoxy-HCN trajectories. The oxygen atom in Oxy-HCN and copper atoms in the Deoxy-HCN were selected as the starting point for tunnel identification. The probe radius was set at $1.0 \AA$ and the clustering threshold value was kept at 10.0 to evaluate cluster results. The tunnels were ranked based on their average bottleneck radius $(\AA)$ and throughput values. The bottleneck radius provides the maximal probe size which can fit in the narrowest part of the tunnel, while the throughput value reflects the probability that the pathway is used as a route for transport of the substances using the formula $e^{- \text {cost }}$, where $e$ is Euler's number and the cost is a function defined as

$$
\int_{0}^{L} r(l)^{-2} d l,
$$

where $L$ is the length of a path, $r(l)$ is a function defining the radius of the largest ball which does not collide with the atoms of the structure and is centered at the point on the pathways axis in the distance $l$ from the starting vertex (Stourac et al., 2019).

\section{Convolutional Variational Autoencoder}

The convolutional variational autoencoder (or CVAE) was implemented for analyzing the simulation trajectories. The CVAE was previously used successfully for multiple different cases, from analyzing protein folding (Bhowmik et al., 2018) to enzyme dynamics (Romero et al., 2019; Akere et al., 2020), to recent COVID-19 related molecular mechanism studies (Acharya et al., 2020; Cho et al., 2021). This specially built CVAE on the HPC platform is able to cluster different microstates of any system based on their subtle difference among 3D structures influenced by various local or global motions (Yoginath et al., 2019).

Essentially, the CVAE architecture is driven by a specific deep learning algorithm. Briefly, the CVAE is built on top of a traditional autoencoder with a variational approach. An autoencoder, in general, has an hourglass-like architecture where the high-dimensional input data is fed. Only the essential information is captured by the autoencoder as the high-dimensional data passes through it. The essential information is then used to reconstruct the original highdimensional data to make sure there is no loss of information during the compression mechanism of the autoencoder. The added variational approach is a key component that optimizes the reduced dimension captured data and forces that to be distributed normally over the latent space, thus ensuring the efficient utilization of the latent space. Simultaneously this also
A

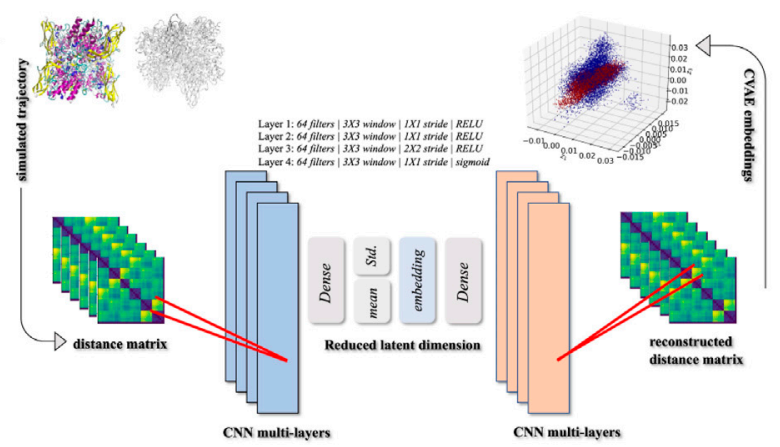

C

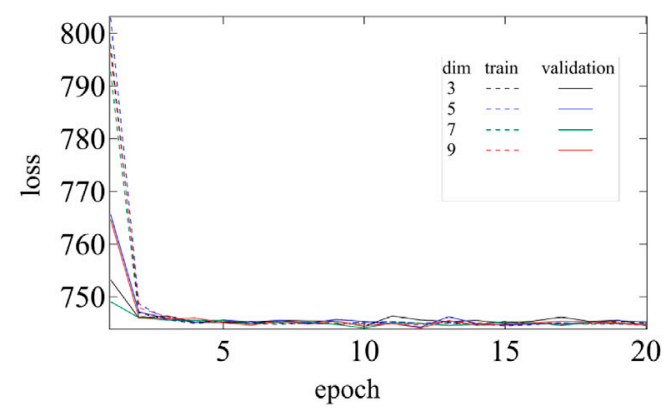

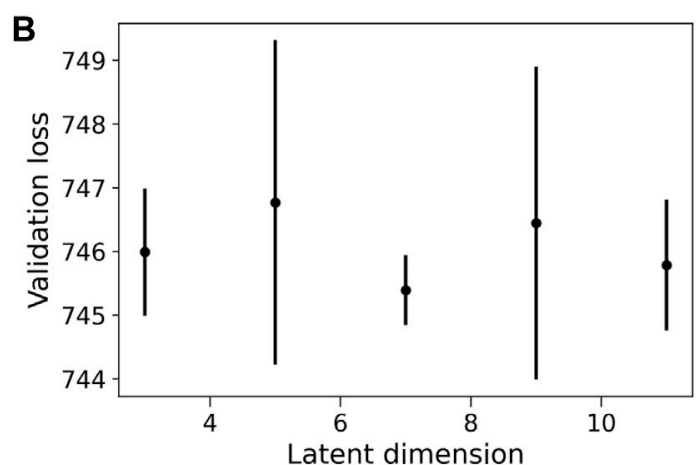

D

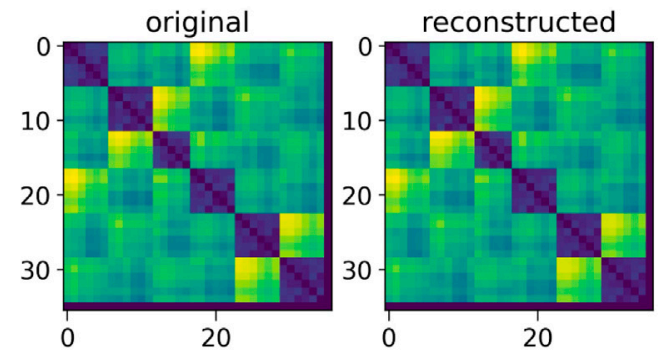

FIGURE 3 | CVAE-based deep learning implementation. (A) The CVAE implementation where distance matrix is used as input data for generating the lowdimensional representations. The input data is initially trained where the quality of training is followed by the different loss values at different dimensions over epochs. (B) The validation loss is shown at different latent dimensions for determining the optimum values of the low dimension. (C) The training and validation loss are assessed over epochs at various dimensions. (D) Comparison between original input data and reconstructed data are shown to ensure no loss of essential information during the compression and reconstruction process 

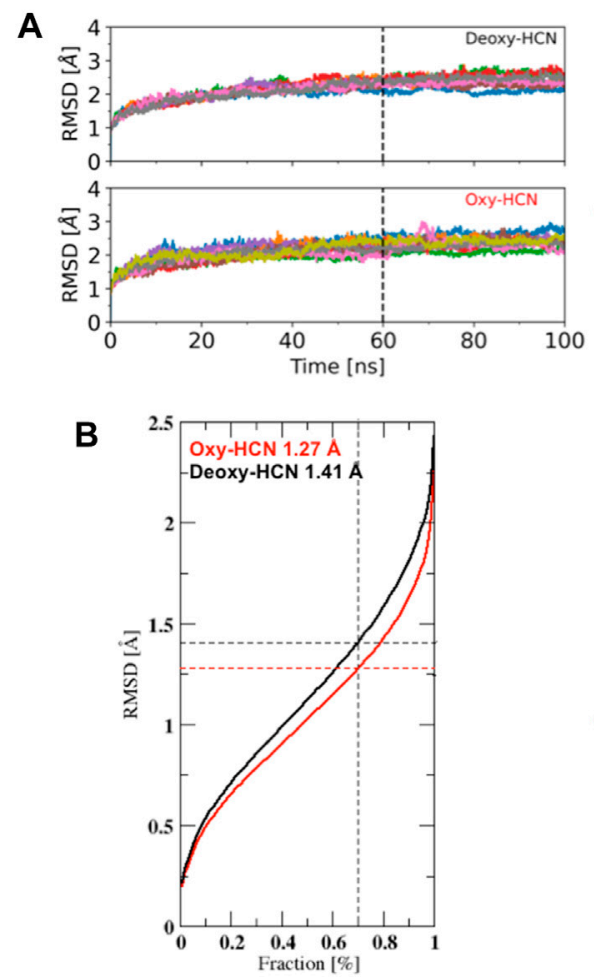
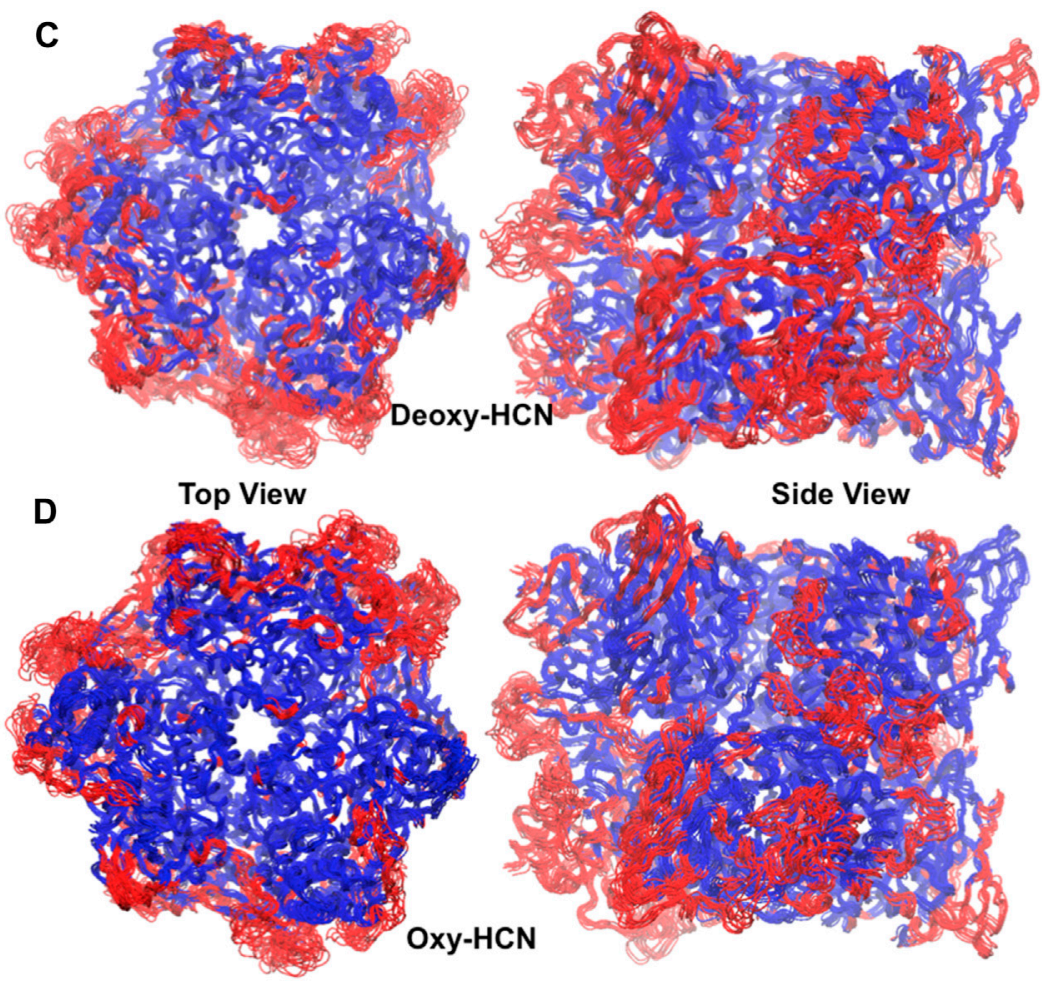

FIGURE 4 | A comparison of Ca conformational drifts between the deoxygenated and oxygenated hemocyanin hexamers. (A) Average Ca RMSD for Deoxy-HCN $(2.55 \AA \pm 0.04)$ and $\mathrm{Oxy}-\mathrm{HCN}(2.43 \AA \pm 0.04)$ are similar when calculated over the last 40 ns equilibrated section of the trajectories. (B) $70 \%$ of Ca atoms in the simulation can be aligned to below 1.41 ̊̊ in Deoxy-HCN (black) and $1.27 \AA$ in Oxy-HCN (red). Top and side views of (C) Oxy-HCN and (D) Deoxy-HCN structures, highlighting the least mobile Ca atoms (70\%) that form the core (blue). The regions of the structure, which are more mobile (red) include the surface loops or short loops that connect different secondary structure elements together.

helps the captured data not to be spreader sparsely and gain the useful knowledge to generate new conformations if required. In the end, the convolutional layers or CNN layers are included so that the local and global information is captured in an efficient way from the multi-layered complex biomolecular structures. The complete schematic CVAE architecture is shown in Figure 3A.

First, the distance matrix of the two systems (i.e., Oxy-HCN and Deoxy-HCN) was built separately using the 36 Histidine residues that coordinate with copper (Figure 2; His172, His177, His204 and His324, His328, His 364). With the parallelized version of the CVAE using the Horovod library, the input distance matrix was directly fed into the CVAE architecture. The training was performed on the Summit supercomputer with a fixed number of epochs. This number is determined by the trade-off between the convergence of loss value and variance bias. The training data were randomly divided into 80:20 ratio for training and validation dataset, respectively. The individual batch size was kept low so that the generalization gap for large-batch size training could be avoided. In order to select the optimal values for clustering parameters with the best reconstruction quality, the training was performed at various latent dimensions. Figure 3B shows how the loss function varies in different dimensions. Comparing the different loss values (Figure 3C) and loss values in different dimensions
(Figure 3B) it is evident that dimension 7 is the best latent dimension to work with. The comparison between the original and reconstructed distance map of the system is also shown in Figure 3D.

\section{RESULTS AND DISCUSSION}

The conformational drift of both systems, Deoxy-HCN and Oxy$\mathrm{HCN}$, were evaluated by calculating the root mean square deviation (RMSD). Conventional methods calculate RMSD by aligning the structure in each frame of the trajectory to a reference structure. This rigid body alignment has a drawback, in the sense that if a small region of the structure is highly flexible, then the RMSD increases for all atoms. This results in an incorrect quantification of the structural deviations and thus poorly assesses important motions associated with biological function. As seen in the case of HCNs, conventional RMSD is unable to differentiate between the dynamics of the two states. The average $\mathrm{Ca}$ RMSD for Deoxy-HCN $(2.55 \AA \pm 0.04)$ and Oxy-HCN $(2.43 \AA \pm 0.04)$ are very similar when calculated over the last $40 \mathrm{~ns}$ of the trajectories (Figure 4A). To overcome this caveat, RMSD was calculated using the MDLovoFit algorithm. This method aligns a fraction of the structure displaying the smallest displacements and enables mobile structures to be 


\section{Deoxy-HCN}
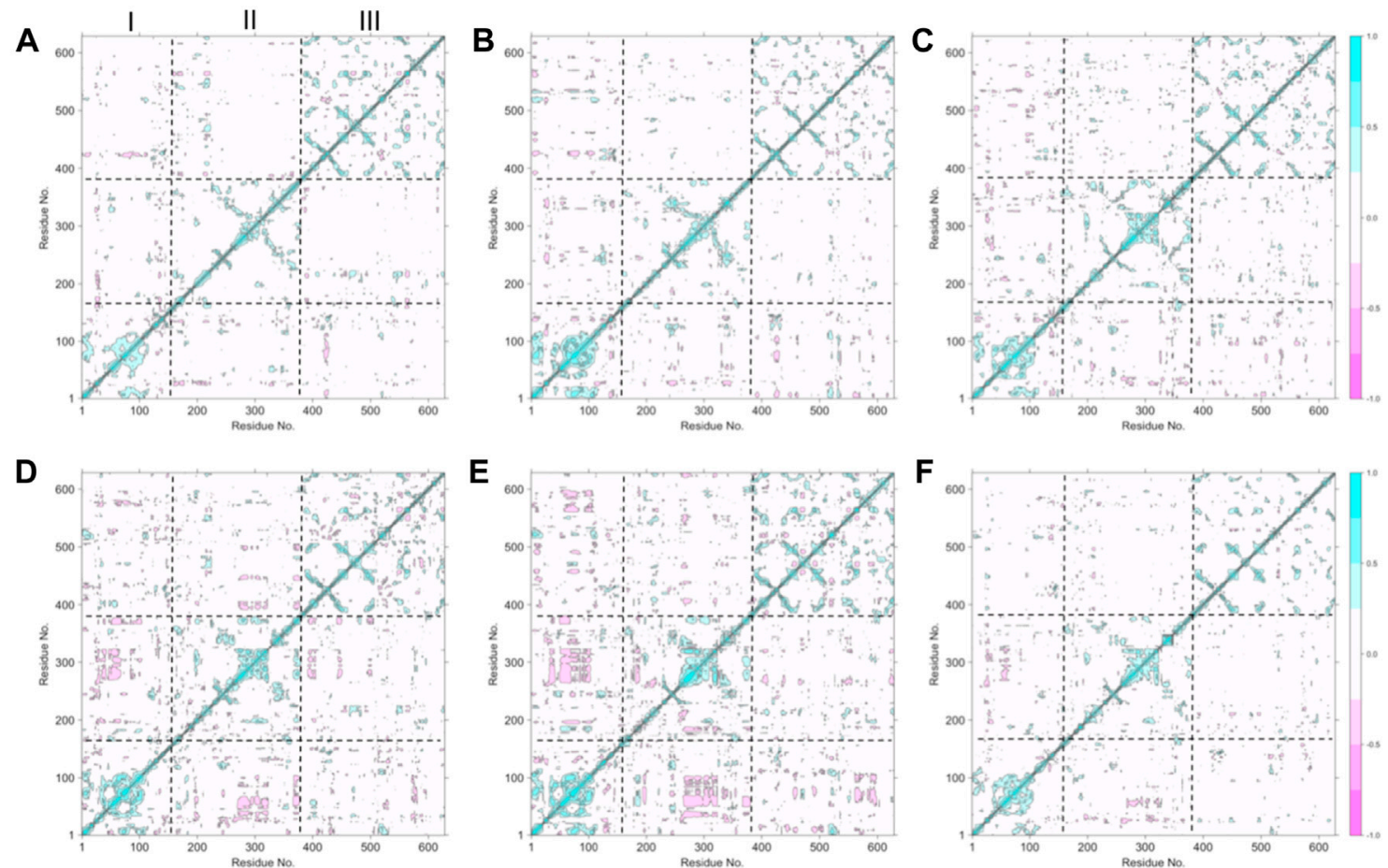

\section{Oxy-HCN}
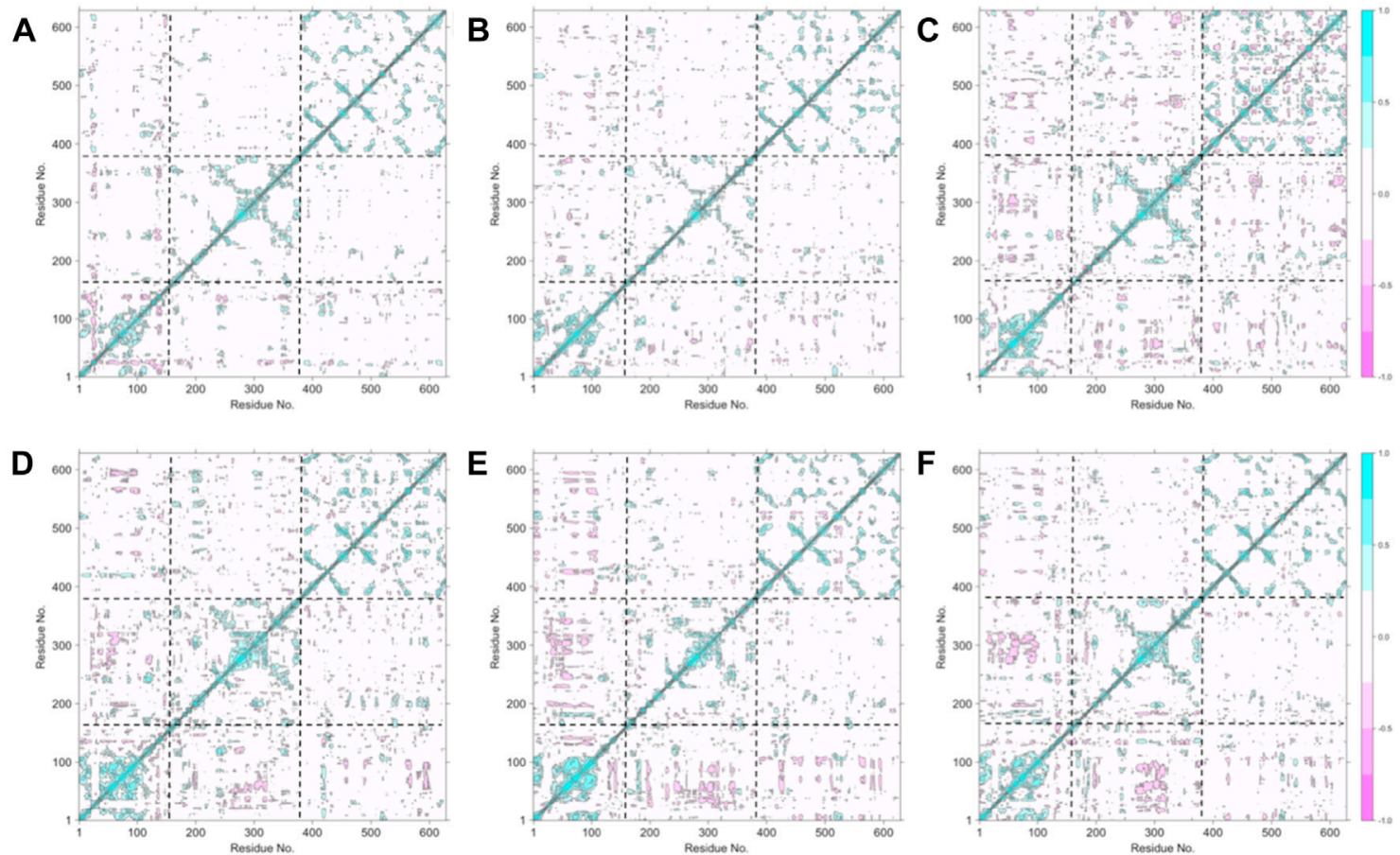

FIGURE 5 | Dynamic cross-correlation matrix (DCCM) maps depicting cross-correlated motion for all six subunits in (A) Deoxy-HCN and (B) Oxy-HCN hexamers. Boundaries of domains I, II, and III are represented by dotted lines. Domains III and I predominantly show positively correlated motions. 

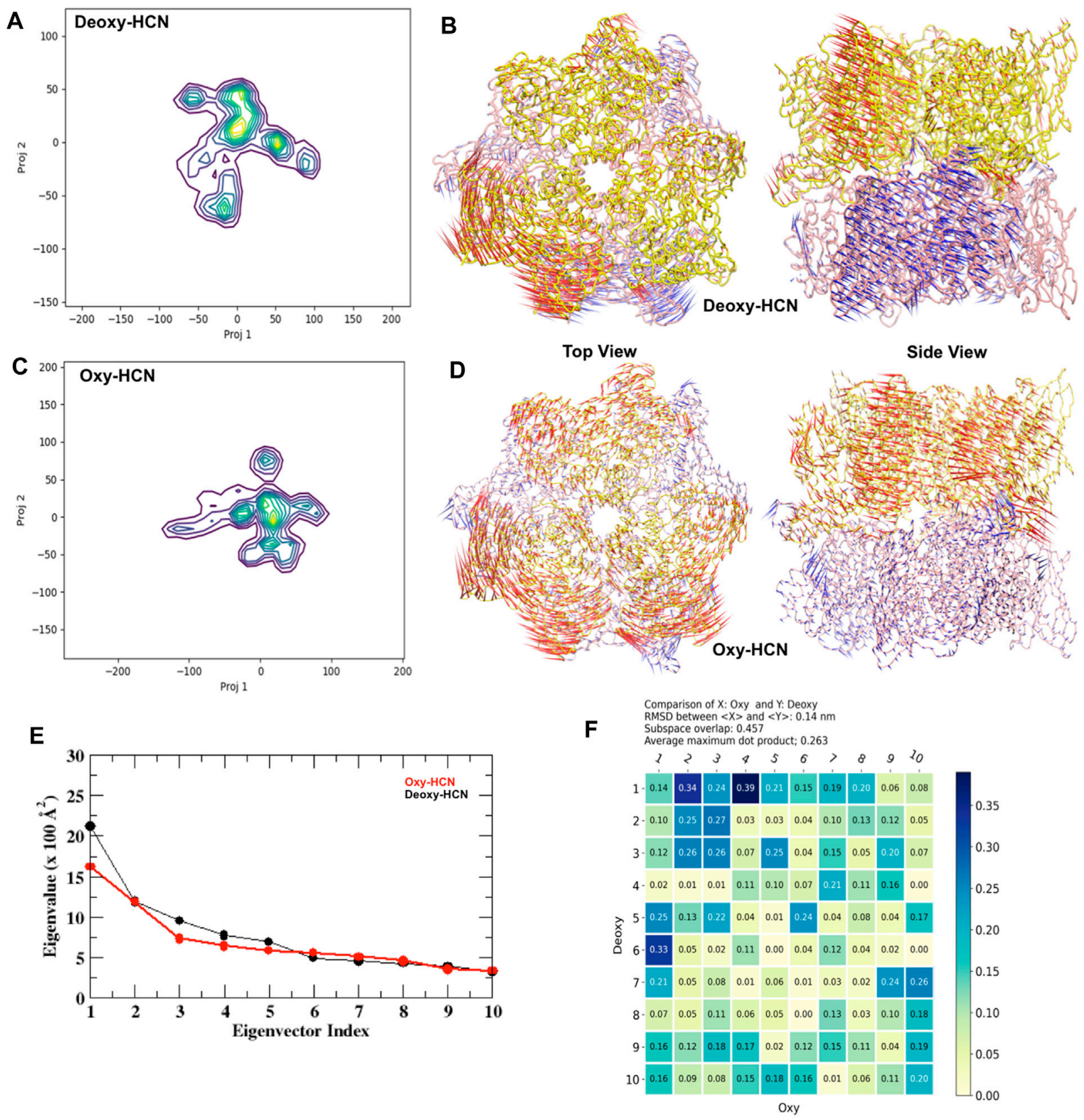

FIGURE 6 | Principal component analysis (PCA). (A) Projections 1 and 2 from Deoxy-HCN represented as a heat map. (B) Top and side view of principal component 1 from Deoxy-HCN. (C) Projections 1 and 2 from Oxy-HCN represented as a heat map. (D) Top and side view of principal component 1 from Deoxy-HCN. (E) A scree plot comparing the eigenvalue versus eigenvector index in the two systems. (F) A subspace analysis between Deoxy-HCN and Oxy-HCN.

identified. It was possible to align $70 \%$ of Deoxy-HCN to below $1.41 \AA$ and Oxy-HCN to $1.27 \AA$ (Figure 4B). The regions of low mobility (blue) are the core of the protein, while the surface loops and loops connecting secondary structure elements contribute to regions of high (red) mobility (Figures 4C,D). The lower RMSD in Oxy-HCN was reasoned to be a result of the collapsed cavities or tunnels due to enhanced inter- and intra-subunit interactions that stabilized the oxygenated state. This is consistent with the experimental finding that the oxygenation process enhances the stability of the hexamer (Sterner et al., 1995; Bux et al., 2018).

The flexibility of both systems was assessed by calculating the root mean square fluctuations (RMSF) of Ca atoms of protein from the last 40 ns of the trajectories (Supplementary Figure S1). Each subunit within the HCN hexamer showed comparable flexibility in both forms of Deoxy- and Oxy-HCN states. Each monomeric unit consists of three domains: domain I comprising 

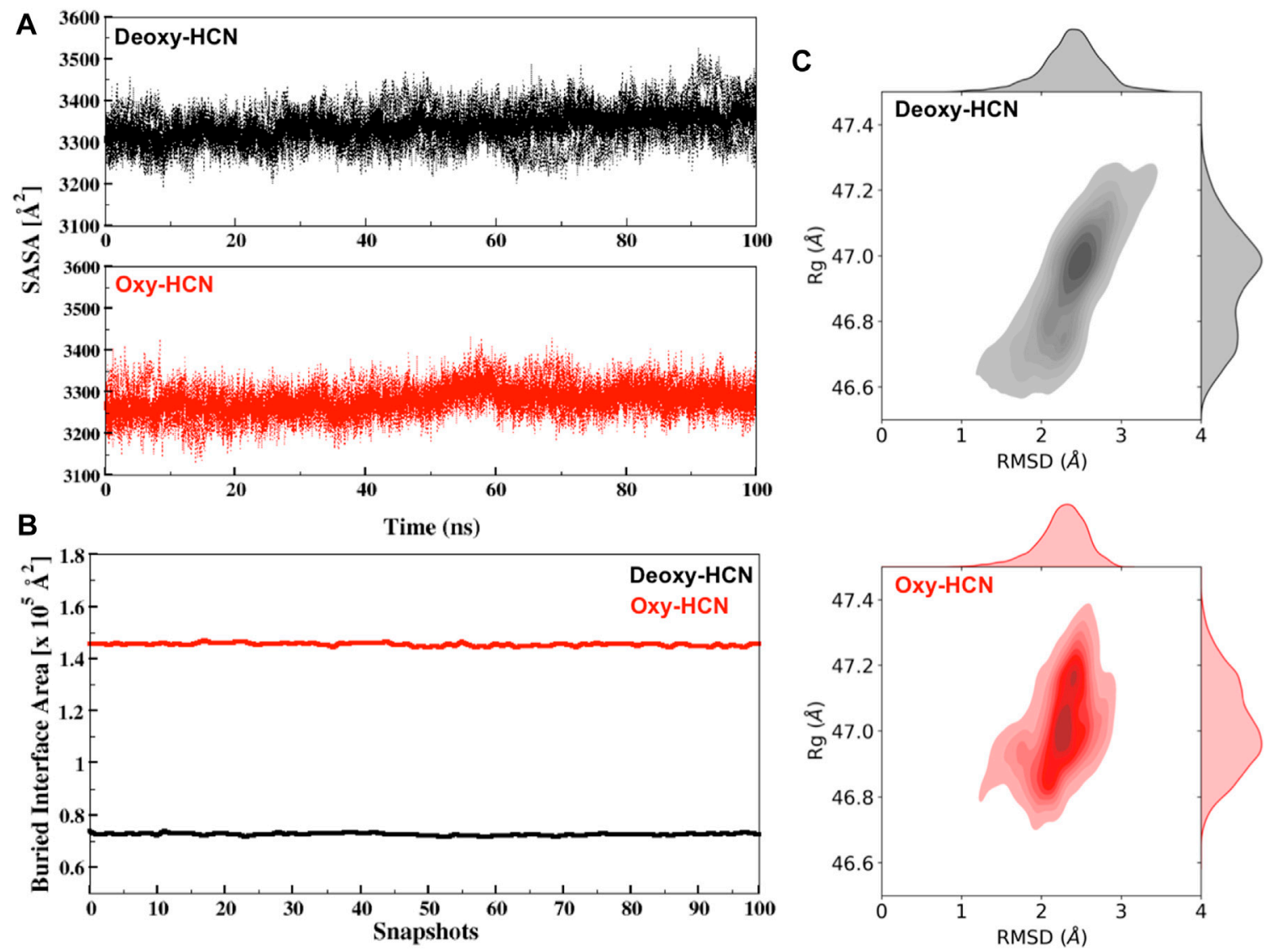

FIGURE 7 | (A) Solvent accessible surface area (SASA) for Deoxy-HCN (black) and Oxy-HCN (red) of all subunits (dashed) and their average (bold). (B) Interface accessible surface area (IASA) buried due to inter-residual contacts for Deoxy-HCN (black) and Oxy-HCN (red) of 100 snapshots extracted from the last 40 ns of each trajectory. (C) The radius of gyration versus Ca-RMSD correlation plot for Deoxy-HCN (black) and Oxy-HCN (red).

amino acid residues from 1 to 154, II that ranges from 155 to 380, and III that contains amino acid residues from 381 to 628 . Of these, domains III and I were found to show more flexibility as compared with domain II in Oxy-HCN. The lower flexibility of domain II was reasoned to be due to the presence of oxygen in the metal binding site within this domain and also due to its spatial position, being sandwiched between domains III and I. In Deoxy$\mathrm{HCN}$ dynamics, three subunits showed an increase in flexibility in domain II, suggesting that residues can be flexible in the absence of oxygen. This observation warranted further investigation into the correlated motions of each domain within the subunits. This was assessed by means of estimating correlated motions across each domain of the subunits individually along with the collective approximation of the individual subunits in the hexameric protein for both Deoxy-HCN and Oxy-HCN systems (Figure 5).

The dynamic cross-correlation maps between Deoxy-HCN and Oxy-HCN are comparable (Figure 5). Domains III and I in each subunit displayed positive correlated motions and smaller anti-correlated motions. Domain II in each subunit displayed more anti-correlated motions. The observations from the dynamic cross-correlation analysis highlight the relationship between oxygenation and associated changes observed between the three domains in the hexameric protein. This is suggestive of the dynamic equilibrium between different conformational states that makes the passage of oxygen possible in each subunit within the hexamer.

Further quantification into the similarities and differences in the global motion of the hexameric protein was assessed using principal component analysis (PCA). PCA was able to extract and filter dominant motions from a set of sampled conformations and define their respective essential space. The top mode was analyzed and is illustrated as a porcupine plot (Figure 6).

The dominant mode (PC1) in Deoxy- $\mathrm{HCN}$ is the rotation of the top trimeric ring with respect to the bottom. The three subunits in the top ring move clockwise and the bottom three anticlockwise. The PC1 in Oxy-HCN is dominated by a clamshell-like movement between two adjacent subunits in each ring (Figure 6). A pairwise comparison between the Deoxy-HCN and Oxy-HCN states was made by calculating the dot product matrix between the eigenvectors identified from PCA of their corresponding states. Such comparison allows quantitative assessment of similarity between the dynamics in the two different systems. Deoxy-HCN and Oxy-HCN simulations have a subspace overlap of $\sim 45.7 \%$ and an average maximum 

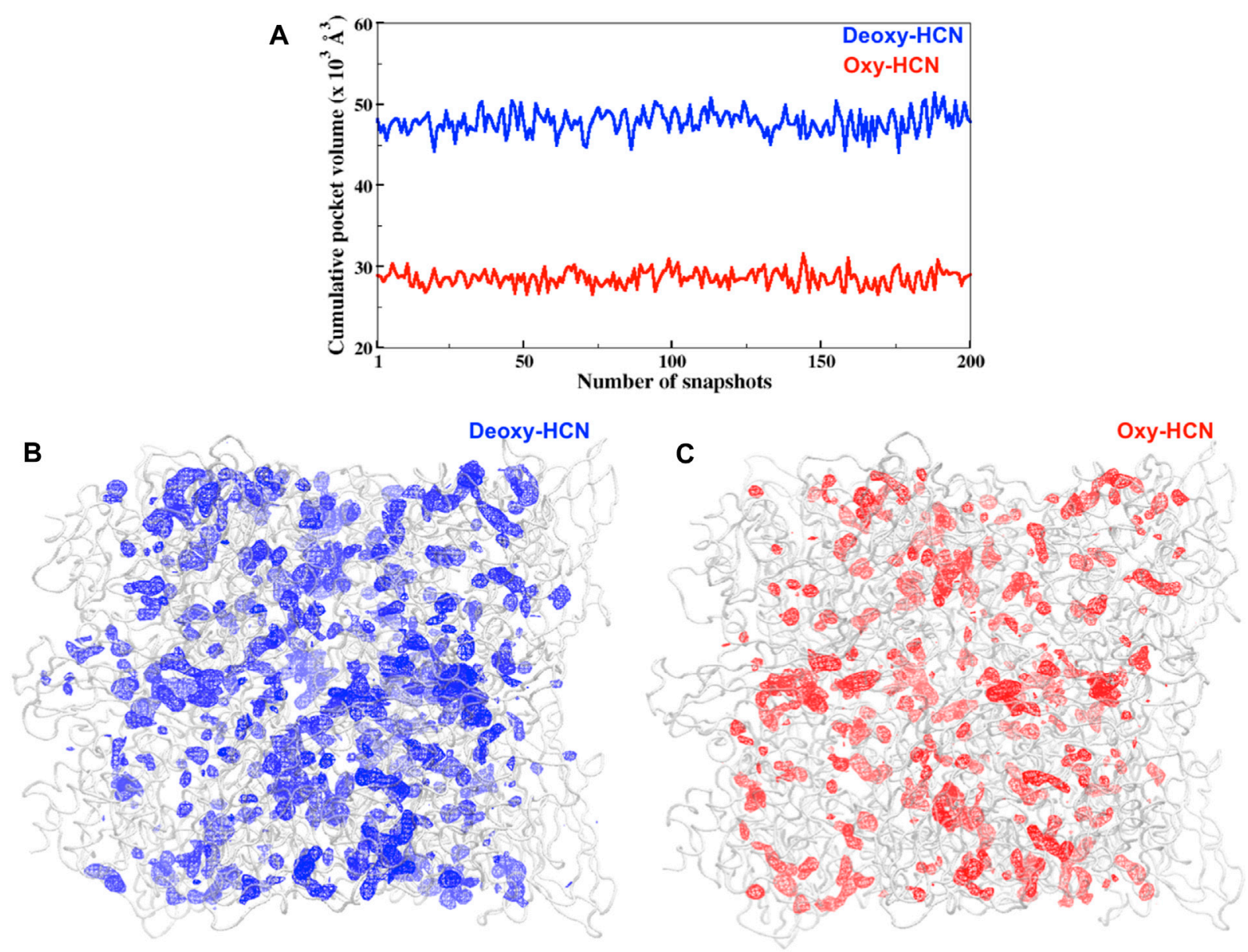

FIGURE 8 | (A) Cumulative pocket volumes of cavities facilitating the passage of oxygen within subunits for Deoxy-HCN (blue) and Oxy-HCN (red). The volume was calculated from 200 snapshots of each system, which was extracted from the last 40 ns of each trajectory (B) Three-dimensional depiction of cavity density (mesh) spread in the (B) Deoxy-HCN (blue) and (C) Oxy-HCN (red) hexamers. The lower cumulative pocket volume Oxy-HCN (red) indicates a more stable closed state in Oxy-HCN.

dot product of 0.26 . The most significant similarity observed between Deoxy-HCN and Oxy-HCN is when comparing PC1 of Deoxy-HCN and PC4 of Oxy-HCN, with an inner dot product of 0.39 (Figure 6). The motion in this PC is dominated by loop interactions at the interface of the two concentric rings. The presented analysis suggests that the Deoxy-HCN and Oxy-HCN simulations occupy only $\sim 50 \%$ of the same conformational (essential dynamics) subspace, though not always sampling the same regions in that space. Such observations from PCA also supported the assumption regarding large-scale dynamic changes when the proteins move from a deoxygenated state to a more stable oxygenated state. Moreover, the variation in the motion of the Deoxy-HCN and Oxy-HCN systems observed in PCA also indicated that the inter-residue contacts at the interface of each subunit might play an important role in the dynamics of the hexamer.

The dynamic states of the hexamer were then assessed by calculating the solvent-accessible surface area (SASA) of each subunit and then averaging it over the hexamer. The changes in SASA were then correlated with the motions to highlight variation in structural dynamics. Deoxy-HCN showed a relatively larger solvent accessible surface area $\left(3337.48 \AA^{2} \pm\right.$ 21.3) as compared to Oxy-HCN $\left(3280 \pm 19.9 \AA^{2}\right)$ hexamer (Figure 7A). The differences between SASA for the two states are very similar to draw any conclusive inferences regarding the dynamics of the systems.

We next analyzed the radius of gyration $(\mathrm{Rg})$ of the hexamer and correlated it with the RMSD (Figure 7C). Rg allows the estimation of the compactness of each system. The Rg values for the two systems are very similar (Deoxy-HCN 47.2 \pm 0.13 ; Oxy-HCN $46.9 \AA \pm 0.16$ ) and the global compactness of the systems is indistinguishable between the two states of the hexameric protein.

To assess interfacial flexibility, the interface area was quantified by measuring the buried interface accessible surface (IASA) that became concealed or buried as a result of interactions taking place among interfacial residues of two trimeric rings of dimer in hexamer (Figure 7B). Oxy-HCN was observed to have relatively larger buried interface area $\left(1.45 \times 10^{5} \AA^{2} \pm 591.9\right)$ as compared to Deoxy-HCN $\left(0.72 \times 10^{5} \AA^{2} \pm 401.6\right)$. A lower value 


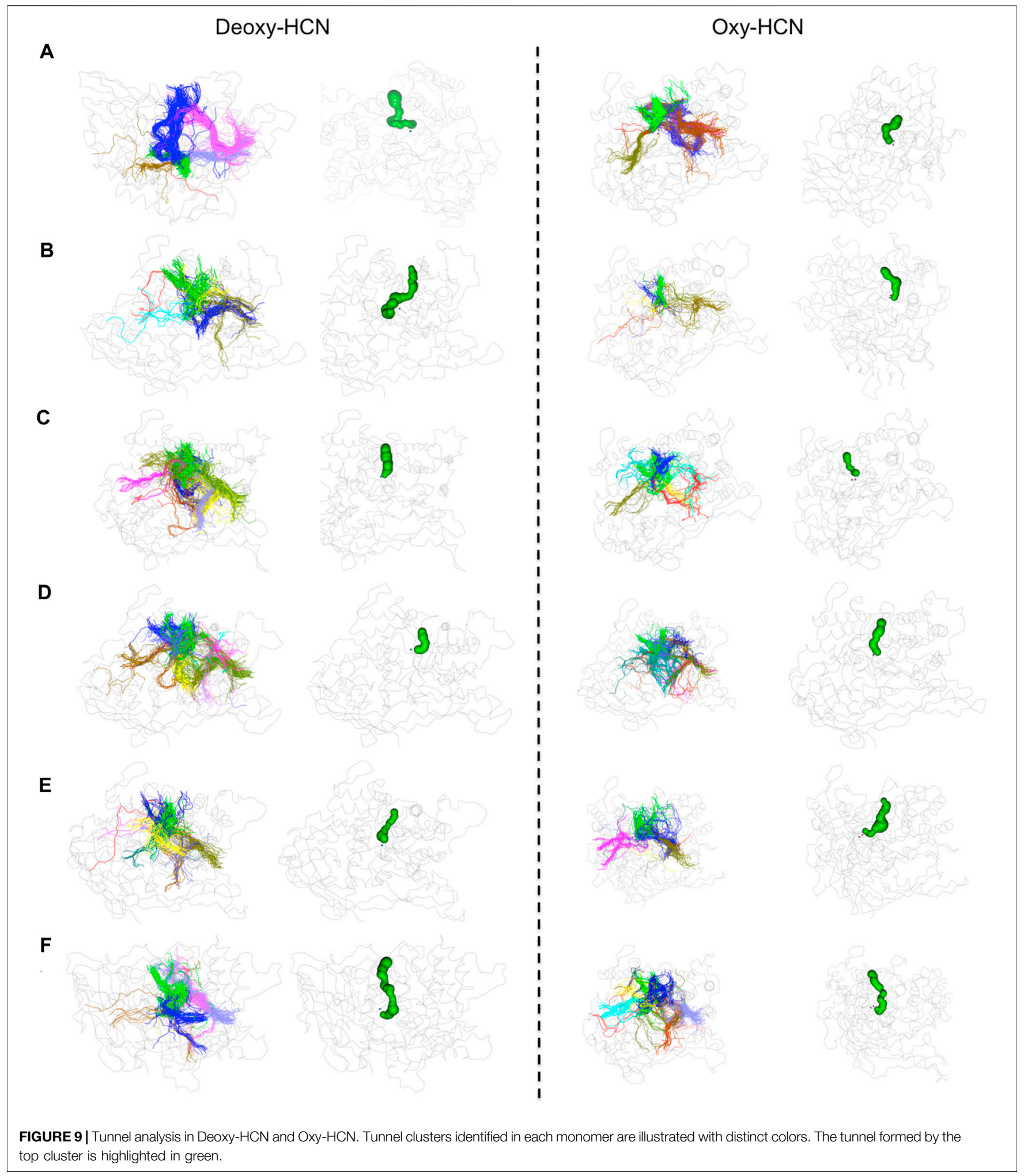

of buried interface in case of Deoxy-HCN suggested more flexibility, which increased the chances of inter residue contacts taking place at the interface of dimer of trimers. In case of Oxy-HCN, a higher value of the buried interface was consistent with the diminished flexibility at the interface and suggested that the dense contacts were a part of the accessible surface area that became concealed or buried in the oxygenated state. 
TABLE 1 | Dynamic tunnel properties of Deoxy- and Oxy-HCN identified by Caver, using a probe radius of $1.0 \AA$ and a clustering threshold of 10.0 (BR: bottleneck radius; TP: throughput).

\begin{tabular}{|c|c|c|c|c|c|c|}
\hline \multicolumn{7}{|c|}{ Oxy-HCN } \\
\hline- & A & B & C & D & $\mathbf{E}$ & $\mathbf{F}$ \\
\hline No. of clusters & 6 & 9 & 8 & 11 & 8 & 12 \\
\hline No. of snapshots & 254 & 37 & 135 & 141 & 111 & 225 \\
\hline Average BR (Å) & 1.20 & 1.10 & 1.24 & 1.09 & 1.14 & 1.19 \\
\hline $\operatorname{Max}$ BR (Å) & 1.50 & 1.31 & 1.82 & 1.72 & 1.54 & 1.71 \\
\hline Average TP & 0.50 & 0.48 & 0.56 & 0.51 & 0.48 & 0.40 \\
\hline \multicolumn{7}{|c|}{ Deoxy-HCN } \\
\hline- & A & B & C & D & $\mathbf{E}$ & $\mathbf{F}$ \\
\hline No. of clusters & 7 & 6 & 10 & 14 & 10 & 11 \\
\hline No. of snapshots & 331 & 101 & 204 & 145 & 95 & 133 \\
\hline Average BR (Å) & 1.33 & 1.14 & 1.21 & 1.20 & 1.20 & 1.15 \\
\hline $\operatorname{Max}$ BR (Å) & 1.93 & 1.60 & 1.71 & 1.73 & 1.72 & 1.60 \\
\hline Average TP & 0.60 & 0.37 & 0.52 & 0.53 & 0.49 & 0.40 \\
\hline
\end{tabular}

Hemocyanins have been reported to have solvent cavities or channels within and at the interface of each subunit (Hazes et al., 1993; Magnus et al., 1994). Thus, investigation of these tunnels or cavities was also carried out to understand their behavior in their respective dynamic states (Figure 8). The cumulative pocket volume calculated in Deoxy-HCN $\left(47.8 \times 10^{3} \AA^{3}\right)$ was greater than that in Oxy-HCN $\left(28.5 \times 10^{3} \AA^{3}\right)$ (Figure 8).

To further validate the observations drawn from the cumulative volume of cavities, dynamic tunnel formation was measured in the simulated systems. A summary of all clusters is tabulated in Table 1. Clusters were calculated for individual subunits within the hexamer and compared between the two states. The identified tunnel clusters are illustrated in Figure 9. The top cluster is the most important due to the higher throughput value and average bottleneck radius. The residues involved in tunnel formation for the top cluster are listed in Table 2. In Deoxy-HCN, 58 cumulative clusters were identified while in Oxy-HCN 54, clusters were observed. For each tunnel cluster, all residues were at least present in one snapshot and within $2 \AA$ from an individual tunnel cluster. The dynamic properties of the tunnel were assessed using a bottleneck analysis. A bottleneck is defined as the narrowest part of the tunnel and the average ranged between 1.1 and $1.2 \AA$ in Oxy$\mathrm{HCN}$. During the course of the dynamics, the constriction formed

TABLE 2 | Tunnel lining residues of the tunnel cluster with the highest average throughput and average bottleneck radius.

\section{OXy-HCN}

A

B

C

$\mathrm{D}$

$E$

E313, D350, T351, S354, L355, F360
F360, W363
C48, F49, P51, L54, F91, W176, Y180, P181, S182, T183, K196, F200, E309, S310, N322, N325, T351, F360

C48, F49, P51, L54, S182, T183, F200, V308, E309, S310, S311, Y312, N322, L323, N325, W326, G327, M348, S354

F49, L54, W176, S182, F200, V308, E309, S310, S311, Y312, N322, N325, T351

C48, F49, H50, P51, L54, H175, W176, S182, T183, F200, E309, S310, S311, Y312, N322, N325, T351, S354, F360

C48, F49, P51, L54, E55, A57, R58, Y61, F91, W174, H175, W176, L178, V179, Y180, P181, S182, T183, W184, K196, F200, E309, N325, D350, T351, S354, F360

C48, F49, W176, Y180, P181, S182, R195, K196, G197, E198, L199, F200, M203, D302, I303, G305, A306, E309, S311,

\section{Deoxy-HCN}

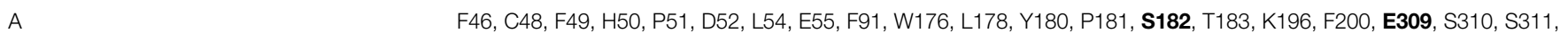
Y312, H317, G321, N322, N325, T351, F360

B

F46, S47, C48, F49, H53, L54, E55, A57, R58, H59, Y61, L88, F91, V95, A171, H172, W174, H175, W176, L178, Y180, P181, S182, T183, K196, F200, M203, M207, E309, N325, T351, S354, L355, F360, W363

C

S47, C48, F49, H50, P51, L54, W176, S182, T183, E309, S310, S311, Y312, H317, N322, S349, D350, T351, S352

D

S47, C48, F49, H50, L54, W174, W176, L178, S182, T183, F200, M203, E309, S310, S311, N322, N325, D350, T351, S352

E

C48, F49, H50, P51, L54, S182, T183, F200, M203, E309, S310, S311, N322, N325, D350, T351, S354, L355, F360

F

F49, H172, W176, V179, Y180, P181, S182, K192, K193, D194, R195, K196, G197, L199, F200, M203, M207, E309 S310, T351, L355, F360, A585, D587, H588, K589, Y590, M596 
A

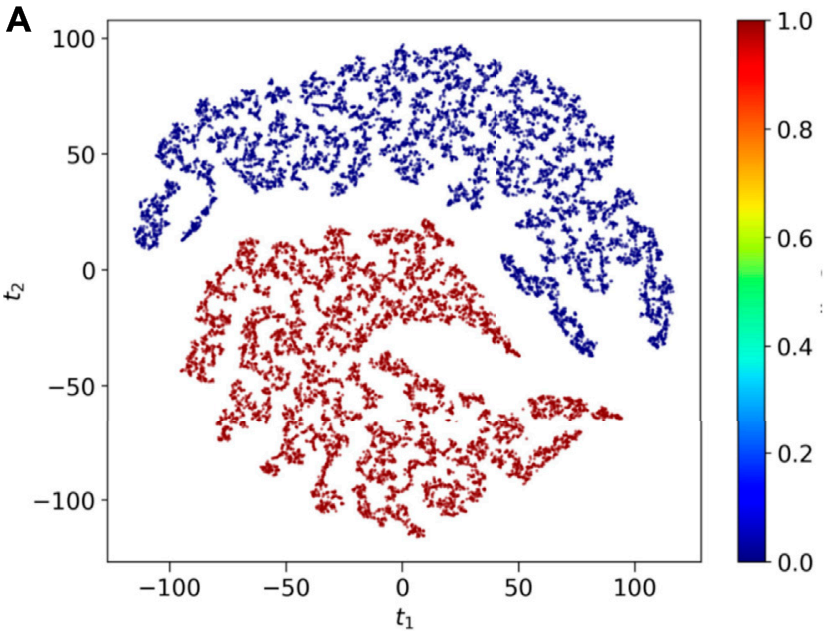

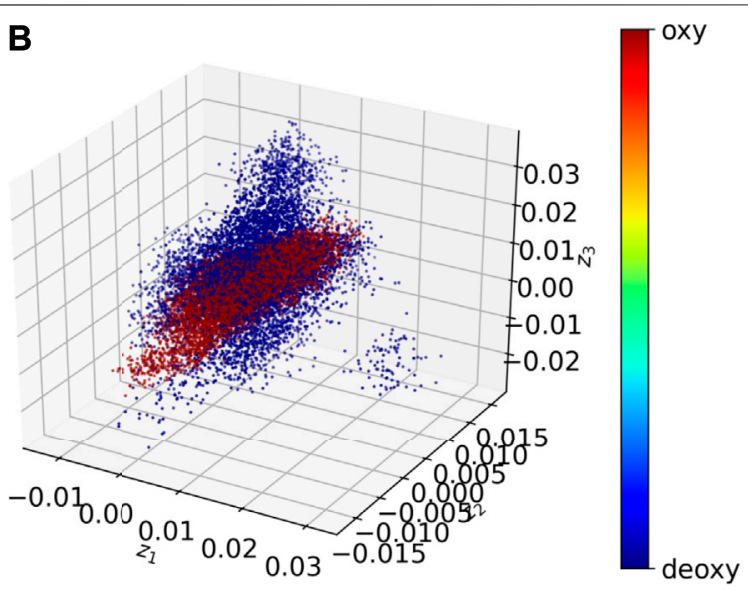

FIGURE 10 I CVAE-based deep learning analysis. The reduced dimensional latent space of CVAE-learnt features of the high dimensional original input are shown in (A) 2D representation and (B) 3D representation following t-sne treatment on the compressed data. The results show that the two systems [i.e., Oxy-HCN (red) and Deoxy-HCN (red)] are different based on their local and global conformational dynamics.

by residues expands from $1.0 \AA$ to $1.8 \AA$, which is enough to allow the oxygen molecule to pass. Similarly in Deoxy-HCN, the bottleneck radius ranged from 1.0 to $1.3 \AA$, while the constriction is able to expand to $1.9 \AA$. In both systems, the top tunnel clusters identified within each monomer share several common residues. Of these, S182, E309, and N325 appear around the bottleneck and contribute towards the constriction of the tunnel. The relatively greater flexibility observed in Deoxy-HCN is in agreement with the conformational state that was ready to facilitate oxygen entry into the protein. Additionally, the cavities and tunnels in the Deoxy-HCN state are correspondingly spread across the interface of all subunits. Smaller and less dense cavities in Oxy-HCN are representative of the conformation of the protein that is acquired after oxygen has passed to the binuclear active site of the protein.

The global dynamics of the Oxy-HCN and Deoxy-HCN are indistinguishable using conventional analysis like RMSD, radius of gyration, and solvent accessible surface area. Thus, a highly sensitive convolutional variational autoencoder (CVAE) based deep learning method was used to differentiate between the dynamics of the two systems. The CVAE model quality was evaluated in different latent dimensions. Dimension 7 turned out to be the best dimension to work with (Figure 3B). The loss behavior over epoch showed a normal trend in all dimensions but the value with the variation in the loss at dimension 7 was better than others (Figure 3B). This is because initially as the latent dimension increases, the model starts compressing less and thus acquires more representation ability. But gradually when the dimensions become too large, then the model might overfit while introducing additional noises. The overall loss attains an optimal value between the two extremes. For Oxy- and DeoxyHCN systems, the CVAE model is quite stable, as the loss stays close to each other in different dimensions. The data was visualized using 2D-tsne ( $\mathrm{t}$-distributed stochastic neighbor embedding) (Figure 10A). CVAE is able to cluster the two systems (i.e., Oxy-HCN (red) and Deoxy-HCN (blue) distinctly based on their local and global conformational dynamics. This is visible both in $2 \mathrm{D}$ - and $3 \mathrm{D}$-dimensional representation (Figure 10B). These results indicate that the dynamics of the oxygenated and deoxygenated systems are discernible.

\section{CONCLUSION}

Molecular dynamics simulation was used to study the differences between the deoxygenated and oxygenated forms of hemocyanin hexamers. This 3768 residue system was assessed over a cumulative sampling time of around $1 \mu$ s (10 replicates of 100 ns each). Conventional structural analysis methods were unable to differentiate between the dynamics of the two systems. This is because the conformations of the starting structure of Oxy-HCN and Deoxy-HCN are very similar. Further investigation of the systems using PCA highlighted less than $\sim 46 \%$ subspace overlap. The most dominant motions identified the hexameric HCN system to function as dimer-of-trimers. The evaluation of interfacial dynamics by estimating the contacts between residues at the interface indicated that the Deoxy-HCN subunits experienced fewer contacts as compared with OxyHCN subunits. Calculations of the interface area via IASA further revealed that the residues at the interface of subunits Deoxy-HCN were less buried than in Oxy-HCN. Estimating the solvent cavities in terms of densities, frequencies and their volumes also found Oxy-HCN to have contracted solvent cavities. The average bottleneck radius of the tunnels identified in the Deoxy-HCN was greater than in Oxy-HCN. The deep learning analysis carried out as a function of the residues that bind to copper in the active site indicates that the two systems are different based on their local and global conformational dynamics. These observations, when taken together confirm a 
conformation for Deoxy-HCN that is ready to accept and transport oxygen to metal binding sites. Once the oxygenation process is completed, the system becomes stable as represented by the Oxy-HCN conformation.

\section{DATA AVAILABILITY STATEMENT}

The raw data supporting the conclusions of this article will be made available by the authors, without undue reservation, to any qualified researcher.

\section{AUTHOR CONTRIBUTIONS}

$\mathrm{KB}$ and $\mathrm{SH}$ designed the study. $\mathrm{KB}, \mathrm{XS}, \mathrm{MT}$, and $\mathrm{SH}$ performed and analyzed the simulations. Deep learning, preprocessing, and related data analysis: JY, DB, and SH. Article writing: KB, SM, DB, and SH. All authors contributed to the article and approved the submitted version.

\section{REFERENCES}

Abraham, M. J., Murtola, T., Schulz, R., Páll, S., Smith, J. C., Hess, B., et al. (2015). GROMACS: High Performance Molecular Simulations through Multi-Level Parallelism from Laptops to Supercomputers. SoftwareX 1-2, 19-25. doi:10.1016/j.softx.2015.06.001

Acharya, A., Agarwal, R., Baker, M. B., Baudry, J., Bhowmik, D., Boehm, S., et al. (2020). Supercomputer-Based Ensemble Docking Drug Discovery Pipeline with Application to Covid-19. J. Chem. Inf. Model. 60, 5832-5852. doi:10.1021/ acs.jcim.0c01010

Akere, A., Chen, S. H., Liu, X., Chen, Y., Dantu, S. C., Pandini, A., et al. (2020). Structure-based Enzyme Engineering Improves Donor-Substrate Recognition of Arabidopsis thaliana Glycosyltransferases. Biochem. J. 477, 2791-2805. doi:10.1042/BCJ20200477

Arancibia, S., Salazar, F., and Becker, M. I. (2012). "Hemocyanins in the Immunotherapy of Superficial Bladder Cancer," in Bladder Cancer-From Basic to Robotic Surgery (Rijeka: InTech), 221-242. doi:10.5772/28281

Balsera, M. A., Wriggers, W., Oono, Y., and Schulten, K. (1996). Principal Component Analysis and Long Time Protein Dynamics. J. Phys. Chem. 100, 2567-2572. doi:10.1021/jp9536920

Bayer, R. (2016). Method of Treating Viral Diseases.

Becker, M. I., Arancibia, S., Salazar, F., Del Campo, M., and De Ioannes, A. (2014). "Mollusk Hemocyanins as Natural Immunostimulants in Biomedical Applications," in Immune Response Activation (Rijeka: InTech), 45-72. doi:10.5772/57552

Berkson, J., and Shuster, C. N., Jr (1999). The Horseshoe Crab: The Battle for a True Multiple-Use Resource. Fisheries 24, 6-10. doi:10.1577/1548-8446(1999) $024<0006$ :thctbf $>2.0$. co $; 2$

Besser, K., Malyon, G. P., Eborall, W. S., Paro da Cunha, G., Filgueiras, J. G., Dowle, A., et al. (2018). Hemocyanin Facilitates Lignocellulose Digestion by woodboring marine Crustaceans. Nat. Commun. 9, 1-14. doi:10.1038/s41467-01807575-2

Bhowmik, D., Gao, S., Young, M. T., and Ramanathan, A. (2018). Deep Clustering of Protein Folding Simulations. BMC Bioinformatics 19, 484. doi:10.1186/ s12859-018-2507-5

Bowers, K. J., Chow, D. E., Xu, H., Dror, R. O., Eastwood, M. P., Gregersen, B. A., et al. (2006). "Scalable Algorithms for Molecular Dynamics Simulations on Commodity Clusters," in SC '06: Proceedings of the 2006 ACM/IEEE Conference on Supercomputing (Tampa, FL, USA: IEEE), 43. doi:10.1109/sc.2006.54

Bux, K., Ali, S. A., and Moin, S. T. (2018). Hydration Facilitates Oxygenation of Hemocyanin: Perspectives from Molecular Dynamics Simulations. Eur. Biophys. J. 47, 925-938. doi:10.1007/s00249-018-1316-0

\section{FUNDING}

This material is based upon work supported by the U.S. Department of Energy, Office of Science, Office of Advanced Scientific Computing Research, under contract number DEAC0500OR22725. This research is sponsored in part by the Laboratory Directed Research and Development Program of Oak Ridge National Laboratory, managed by UT-Battelle, LLC, for the U.S. Department of Energy. This research used resources of the Oak Ridge Leadership Computing Facility at the Oak Ridge National Laboratory, which is supported by the Office of Science of the U.S. Department of Energy under Contract no. DE-AC05-00OR22725.

\section{SUPPLEMENTARY MATERIAL}

The Supplementary Material for this article can be found online at https://www.frontiersin.org/articles/10.3389/fmolb.2021.710623/ full\#supplementary-material

Cerutti, D. S., Duke, R. E., Darden, T. A., and Lybrand, T. P. (2009). Staggered Mesh Ewald: an Extension of the Smooth Particle-Mesh Ewald Method Adding Great Versatility. J. Chem. Theor. Comput. 5, 2322-2338. doi:10.1021/ct9001015

Chen, J., Sawyer, N., and Regan, L. (2013). Protein-protein Interactions: General Trends in the Relationship between Binding Affinity and Interfacial Buried Surface Area. Protein Sci. 22, 510-515. doi:10.1002/pro.2230

Cho, E., Rosa, M., Anjum, R., Mehmood, S., Soban, M., Mujtaba, M., et al. (2021). Dynamic Profiling of $\beta$-Coronavirus 3CL $M{ }^{\text {pro }}$ Protease Ligand-Binding Sites. J. Chem. Inf. Model. 61 (6), 3058-3073. doi:10.1021/acs.jcim.1c00449

Chovancova, E., Pavelka, A., Benes, P., Strnad, O., Brezovsky, J., Kozlikova, B., et al. (2012). CAVER 3.0: A Tool for the Analysis of Transport Pathways in Dynamic Protein Structures. Plos Comput. Biol. 8, e1002708. doi:10.1371/ journal.pcbi.1002708

Coates, C. J., Kelly, S. M., and Nairn, J. (2011). Possible Role of PhosphatidylserineHemocyanin Interaction in the Innate Immune Response of Limulus polyphemus. Dev. Comp. Immunol. 35, 155-163. doi:10.1016/j.dci.2010.08.015

Coates, C. J., and Nairn, J. (2014). Diverse Immune Functions of Hemocyanins. Dev. Comp. Immunol. 45, 43-55. doi:10.1016/j.dci.2014.01.021

Coates, C. J., and Nairn, J. (2013). Hemocyanin-derived Phenoloxidase Activity: a Contributing Factor to Hyperpigmentation in Nephrops norvegicus. Food Chem. 140, 361-369. doi:10.1016/j.foodchem.2013.02.072

Coates, C. J., and Talbot, J. (2018). Hemocyanin-derived Phenoloxidase Reaction Products Display Anti-infective Properties. Dev. Comp. Immunol. 86, 47-51. doi:10.1016/j.dci.2018.04.017

Decker, H., Connelly, P. R., Robert, C. H., and Gill, S. J. (1988). Nested Allosteric Interaction in Tarantula Hemocyanin Revealed through the Binding of Oxygen and Carbon Monoxide. Biochemistry 27, 6901-6908. doi:10.1021/bi00418a036

Decker, H., and Tuczek, F. (2000). Tyrosinase/catecholoxidase Activity of Hemocyanins: Structural Basis and Molecular Mechanism. Trends Biochemical Sciences 25, 392-397. doi:10.1016/s0968-0004(00)01602-9

Del Campo, M., Arancibia, S., Nova, E., Salazar, F., González, A., Moltedo, B., et al (2011). [Hemocyanins as Immunostimulants]. Rev. Med. Chil 139, 236-246.

Dolashka, P., Dolashki, A., Van Beeumen, J., Floetenmeyer, M., Velkova, L., Stevanovic, S., et al. (2016). Antimicrobial Activity of Molluscan Hemocyanins from Helix and Rapana Snails. Cpb 17, 263-270. doi:10.2174/ 1389201016666150907113435

Fariselli, P., Bottoni, A., Bernardi, F., and Casadio, R. (1999). Quantum Mechanical Analysis of Oxygenated and Deoxygenated States of Hemocyanin: Theoretical Clues for a Plausible Allosteric Model of Oxygen Binding. Protein Sci. 8, 1546-1550. doi:10.1110/ps.8.7.1546

Fellers, C. R., and Harris, S. G. (1940). Canned Atlantic Crab Meat A New American Food. Ind. Eng. Chem. 32, 592-594. doi:10.1021/ie50364a033 
Gesheva, V., Chausheva, S., Mihaylova, N., Manoylov, I., Doumanova, L., Idakieva, K., et al. (2014). Anti-cancer Properties of Gastropodan Hemocyanins in Murine Model of colon Carcinoma. BMC Immunol. 15, 34. doi:10.1186/ s12865-014-0034-3

Gibson, D. G., III, and Hilly, J. B. (1992). Production of Horseshoe Crab Amebocytes in Vitro.

Grant, B. J., Rodrigues, A. P. C., ElSawy, K. M., McCammon, J. A., and Caves, L. S. D. (2006). Bio3d: an R Package for the Comparative Analysis of Protein Structures. Bioinformatics 22, 2695-2696. doi:10.1093/bioinformatics/btl461

Guncheva, M., Stoyanova, E., Todinova, S., and Idakieva, K. (2019). Chemically Modified Hemocyanins with Enhanced Antibreast Cancer Activities. Proceedings 22, 13. doi:10.3390/proceedings2019022013

Haider, S., Parkinson, G. N., and Neidle, S. (2008). Molecular Dynamics and Principal Components Analysis of Human Telomeric Quadruplex Multimers. Biophysical J. 95, 296-311. doi:10.1529/biophysj.107.120501

Hanyecz, A., Berlo, S. E., Szántó, S., Broeren, C. P. M., Mikecz, K., and Glant, T. T. (2004). Achievement of a Synergistic Adjuvant Effect on Arthritis Induction by Activation of Innate Immunity and Forcing the Immune Response toward the Th1 Phenotype. Arthritis Rheum. 50, 1665-1676. doi:10.1002/art.20180

Harris, J. R., and Markl, J. (1999). Keyhole Limpet Hemocyanin (KLH): a Biomedical Review. Micron 30, 597-623. doi:10.1016/s0968-4328(99)00036-0

Hazes, B., Kalk, K. H., Hol, W. G. J., Magnus, K. A., Bonaventura, C., Bonaventura, J., et al. (1993). Crystal Structure of Deoxygenated Limulus polyphemus Subunit II Hemocyanin at $2.18 \AA$ Resolution: Clues for a Mechanism for Allosteric Regulation. Protein Sci. 2, 597-619. doi:10.1002/pro.5560020411

Himmelwright, R. S., Eickman, N. C., LuBien, C. D., and Solomon, E. I. (1980). Chemical and Spectroscopic Comparison of the Binuclear Copper Active Site of Mollusc and Arthropod Hemocyanins. J. Am. Chem. Soc. 102, 5378-5388. doi:10.1021/ja00536a044

Himmelwright, R. S., Eickman, N. C., and Solomon, E. I. (1978). Preparation and Characterization of Met Apo Hemocyanin: A Single Copper(II) Active Site. Biochem. biophysical Res. Commun. 81, 243-247. doi:10.1016/0006-291x(78) 91656-x

Hornak, V., Abel, R., Okur, A., Strockbine, B., Roitberg, A., and Simmerling, C. (2006). Comparison of Multiple Amber Force fields and Development of Improved Protein Backbone Parameters. Proteins 65, 712-725. doi:10.1002/ prot.21123

Hu, Z., Williams, R. D., Tran, D., Spiro, T. G., and Gorun, S. M. (2000). Reengineering Enzyme-Model Active Sites: Reversible Binding of Dioxygen at Ambient Conditions by a Bioinspired Copper Complex. J. Am. Chem. Soc. 122, 3556-3557. doi:10.1021/ja992017r

Hubbard, S. J., and Thornton, J. M. (1993). NACCESS. Computer Program, Department of Biochemistry and Molecular Biology, University College London. London: Scientific Research Publishing Inc., 2.

Humphrey, W., Dalke, A., and Schulten, K. (1996). VMD: Visual Molecular Dynamics. J. Mol. Graph 14 (33-38), 33-38. doi:10.1016/0263-7855(96) 00018-5

Hundahl, C., Fago, A., and Weber, R. E. (2003). Effects of Water Activity on Oxygen-Binding in High-Molecular Weight, Extracellular Invertebrate Hemoglobin and Hemocyanin. Comp. Biochem. Physiol. B: Biochem. Mol. Biol. 136, 83-90. doi:10.1016/s1096-4959(03)00176-3

Jaenicke, E., Pairet, B., Hartmann, H., and Decker, H. (2012). Crystallization and Preliminary Analysis of Crystals of the 24-meric Hemocyanin of the Emperor Scorpion (Pandinus Imperator). PLoS One 7, e32548. doi:10.1371/ journal.pone. 0032548

Karplus, M., and McCammon, J. A. (2002). Molecular Dynamics Simulations of Biomolecules. Nat. Struct. Biol. 9, 646-652. doi:10.1038/nsb0902-646

Kasahara, K., Fukuda, I., and Nakamura, H. (2014). A Novel Approach of Dynamic Cross Correlation Analysis on Molecular Dynamics Simulations and its Application to Ets1 Dimer-DNA Complex. PLoS One 9, e112419. doi:10.1371/journal.pone.0112419

Kräutler, V., Van Gunsteren, W. F., and Hünenberger, P. H. (2001). A Fast SHAKE Algorithm to Solve Distance Constraint Equations for Small Molecules in Molecular Dynamics Simulations. J. Comput. Chem. 22, 501-508.

Krisfalusi-Gannon, J., Ali, W., Dellinger, K., Robertson, L., Brady, T. E., Goddard, M. K. M., et al. (2018). The Role of Horseshoe Crabs in the Biomedical Industry and Recent Trends Impacting Species Sustainability. Front. Mar. Sci. 5, 185. doi:10.3389/fmars.2018.00185
Kumar, V., Roy, S., Sahoo, A. K., Behera, B. K., and Sharma, A. P. (2015). Horseshoe Crab and its Medicinal Values.

Lei, K., Li, F., Zhang, M., Yang, H., Luo, T., and Xu, X. (2008). Difference between Hemocyanin Subunits from Shrimp Penaeus Japonicus in Anti-WSSV Defense. Dev. Comp. Immunol. 32, 808-813. doi:10.1016/j.dci.2007.11.010

Levin, J., Tomasulo, P. A., and Oser, R. S. (1970). Detection of Endotoxin in Human Blood and Demonstration of an Inhibitor. J. Lab. Clin. Med. 75, 903-911.

Levin, J., and Bang, F. B. (1968). Clottable Protein in Limulus: Its Localization and Kinetics of its Coagulation by Endotoxin. Thromb. Haemost. 19, 186-197. doi:10.1055/s-0038-1651195

Ma, J. (2005). Usefulness and Limitations of normal Mode Analysis in Modeling Dynamics of Biomolecular Complexes. Structure 13, 373-380. doi:10.1016/ j.str.2005.02.002

Magnus, K. A., Hazes, B., Ton-That, H., Bonaventura, C., Bonaventura, J., and Hol, W. G. J. (1994). Crystallographic Analysis of Oxygenated and Deoxygenated States of Arthropod Hemocyanin Shows Unusual Differences. Proteins 19, 302-309. doi:10.1002/prot.340190405

Magnus, K. A., and Ton-That, H. (1991). The crystal Structure of the Oxygenated Form of Subunit II of Limulus polyphemus Hemocyanin. J. Inorg. Biochem. 43, 167. doi:10.1016/0162-0134(91)84159-7

Maloney, T., Phelan, R., and Simmons, N. (2018). Saving the Horseshoe Crab: A Synthetic Alternative to Horseshoe Crab Blood for Endotoxin Detection. Plos Biol. 16, e2006607. doi:10.1371/journal.pbio.2006607

Marsh, J. A., and Teichmann, S. A. (2011). Relative Solvent Accessible Surface Area Predicts Protein Conformational Changes upon Binding. Structure 19, 859-867. doi:10.1016/j.str.2011.03.010

Martínez, L. (2015). Automatic Identification of Mobile and Rigid Substructures in Molecular Dynamics Simulations and Fractional Structural Fluctuation Analysis. PLoS ONE 10, e0119264. doi:10.1371/journal.pone.0119264

Martínez-Rosell, G., Giorgino, T., and De Fabritiis, G. (2017). PlayMolecule ProteinPrepare: A Web Application for Protein Preparation for Molecular Dynamics Simulations. J. Chem. Inf. Model. 57, 1511-1516. doi:10.1021/ acs.jcim.7b00190

Marxen, J. C., Pick, C., Oakley, T. H., and Burmester, T. (2014). Occurrence of Hemocyanin in Ostracod Crustaceans. J. Mol. Evol. 79, 3-11. doi:10.1007/ s00239-014-9636-x

Matsushima, H., Hayashi, S., and Shimada, S. (2003). Skin Scratching Switches Immune Responses from Th2 to Th1 Type in Epicutaneously Immunized Mice. J. Dermatol. Sci. 32, 223-230. doi:10.1016/s0923-1811(03)00106-3

Mazzotti, F., Beuttler, J., Zeller, R., Fink, U., Schindler, S., Wendel, A., et al. (2007). In Vitro pyrogen Test-A New Test Method for Solid Medical Devices. J. Biomed. Mater. Res. 80A, 276-282. doi:10.1002/jbm.a.30922

Miles, D., Roché, H., Martin, M., Perren, T. J., Cameron, D. A., Glaspy, J., et al. (2011). Phase III Multicenter Clinical Trial of the Sialyl-TN (STn)-Keyhole Limpet Hemocyanin (KLH) Vaccine for Metastatic Breast Cancer. The oncologist 16, 1092-1100. doi:10.1634/theoncologist.2010-0307

Mora Román, J. J., Del Campo, M., Villar, J., Paolini, F., Curzio, G., Venuti, A., et al. (2019). Immunotherapeutic Potential of Mollusk Hemocyanins in Combination with Human Vaccine Adjuvants in Murine Models of Oral Cancer. J. Immunol. Res. 2019, 7076942. doi:10.1155/2019/7076942

Musselli, C., Livingston, P. O., and Ragupathi, G. (2001). Keyhole Limpet Hemocyanin Conjugate Vaccines against Cancer: the Memorial Sloan Kettering Experience. J. Cancer Res. Clin. Oncol. 127, R20-R26. doi:10.1007/ bf01470995

Naresh, K. N., Sreekumar, A., and Rajan, S. S. (2015). Structural Insights into the Interaction between Molluscan Hemocyanins and Phenolic Substrates: an In Silico Study Using Docking and Molecular Dynamics. J. Mol. Graphics Model. 61, 272-280. doi:10.1016/j.jmgm.2015.07.006

Neutze, R. (2014). Opportunities and Challenges for Time-Resolved Studies of Protein Structural Dynamics at X-ray Free-Electron Lasers. Phil. Trans. R. Soc. B 369, 20130318. doi:10.1098/rstb.2013.0318

Odell, J., Mather, M. E., and Muth, R. M. (2005). A Biosocial Approach for Analyzing Environmental Conflicts: a Case Study of Horseshoe Crab Allocation. Bioscience 55, 735-748. doi:10.1641/0006-3568(2005)055[0735: abafae $2.0 . c 0 ; 2$

Ohtaki, H., and Radnai, T. (1993). Structure and Dynamics of Hydrated Ions. Chem. Rev. 93, 1157-1204. doi:10.1021/cr00019a014 
Owings, M., Chabot, C., and Watson, W., III (2019). Effects of the Biomedical Bleeding Process on the Behavior of the American Horseshoe Crab,Limulus polyphemus, in its Natural Habitat. Biol. Bull. 236, 207-223. doi:10.1086/ 702917

Pettersen, E. F., Goddard, T. D., Huang, C. C., Couch, G. S., Greenblatt, D. M., Meng, E. C., et al. (2004). UCSF Chimera?A Visualization System for Exploratory Research and Analysis. J. Comput. Chem. 25, 1605-1612. doi:10.1002/jcc.20084

Pick, C., Hagnerholler, S., and Burmester, T. (2008). Molecular Characterization of Hemocyanin and Hexamerin from the Firebrat Thermobia domestica (Zygentoma). Insect Biochem. Mol. Biol. 38, 977-983. doi:10.1016/ j.ibmb.2008.08.001

Pinnow, P., Fabrizius, A., Pick, C., and Burmester, T. (2016). Identification and Characterisation of Hemocyanin of the Fish Louse Argulus (Crustacea: Branchiura). J. Comp. Physiol. B 186, 161-168. doi:10.1007/s00360-0150943-3

Prosser, C. L., and Brown, F. (1961). Comparative Animal Physiology. Philadelphia, PA.: WB Saunders.

Qin, Z., Babu, V. S., Wan, Q., Muhammad, A., Li, J., Lan, J., et al. (2018). Antibacterial Activity of Hemocyanin from Red Swamp Crayfish (Procambarus clarkii). Fish Shellfish Immunol. 75, 391-399. doi:10.1016/j.fsi.2018.02.010

Rehm, P., Pick, C., Borner, J., Markl, J., and Burmester, T. (2012). The Diversity and Evolution of Chelicerate Hemocyanins. BMC Evol. Biol. 12, 19. doi:10.1186/ 1471-2148-12-19

Riggs, D. R., Jackson, B., Vona-Davis, L., and McFadden, D. (2002). In Vitro anticancer Effects of a Novel Immunostimulant: Keyhole Limpet Hemocyanin. J. Surg. Res. 108, 279-284. doi:10.1006/jsre.2002.6548

Roe, D. R., and Cheatham, T. E. (2013). PTRAJ and CPPTRAJ: Software for Processing and Analysis of Molecular Dynamics Trajectory Data. J. Chem. Theor. Comput. 9, 3084-3095. doi:10.1021/ct400341p

Romero, R., Ramanathan, A., Yuen, T., Bhowmik, D., Mathew, M., Munshi, L. B., et al. (2019). Mechanism of Glucocerebrosidase Activation and Dysfunction in Gaucher Disease Unraveled by Molecular Dynamics and Deep Learning. Proc. Natl. Acad. Sci. USA. 116, 5086-5095. doi:10.1073/pnas.1818411116

Rutecki, D., Carmichael, R. H., and Valiela, I. (2004). Magnitude of Harvest of Atlantic Horseshoe crabs,Limulus polyphemus, in Pleasant Bay, Massachusetts. Estuaries 27, 179-187. doi:10.1007/bf02803374

Sagui, C., and Darden, T. A. (1999). Molecular Dynamics Simulations of Biomolecules: Long-Range Electrostatic Effects. Annu. Rev. Biophys. Biomol. Struct. 28, 155-179. doi:10.1146/annurev.biophys.28.1.155

Saito, T., and Thiel, W. (2014). Quantum Mechanics/molecular Mechanics Study of Oxygen Binding in Hemocyanin. J. Phys. Chem. B 118, 5034-5043. doi:10.1021/jp5003885

Schmidtke, P., Bidon-Chanal, A., Luque, F. J., and Barril, X. (2011). MDpocket: Open-Source Cavity Detection and Characterization on Molecular Dynamics Trajectories. Bioinformatics 27, 3276-3285. doi:10.1093/bioinformatics/btr550

Shkurti, A., Goni, R., Andrio, P., Breitmoser, E., Bethune, I., Orozco, M., et al. (2016). pyPcazip: A PCA-Based Toolkit for Compression and Analysis of Molecular Simulation Data. SoftwareX 5, 44-50. doi:10.1016/j.softx.2016.04.002

Sterner, R., Vogl, T., Hinz, H.-J., Penz, F., Hoff, R., Föll, R., et al. (1995). Extreme Thermostability of Tarantula Hemocyanin. FEBS Lett. 364, 9-12. doi:10.1016/ 0014-5793(95)00341-6
Stourac, J., Vavra, O., Kokkonen, P., Filipovic, J., Pinto, G., Brezovsky, J., et al. (2019). Caver Web 1.0: Identification of Tunnels and Channels in Proteins and Analysis of Ligand Transport. Nucleic Acids Res. 47, W414-W422. doi:10.1093/ nar/gkz378

T., B. (2002). Origin and Evolution of Arthropod Hemocyanins and Related Proteins. J. Comp. Physiol. B: Biochem. Systemic, Environ. Physiol. 172, 95-107. doi:10.1007/s00360-001-0247-7

Terwilliger, N. B. (1998). Functional Adaptations of Oxygen-Transport Proteins. J. Exp. Biol. 201, 1085-1098. doi:10.1242/jeb.201.8.1085

Tomasello, G., Armenia, I., and Molla, G. (2020). The Protein Imager: a FullFeatured Online Molecular Viewer Interface with Server-Side HQ-Rendering Capabilities. Bioinformatics 36, 2909-2911. doi:10.1093/bioinformatics/ btaa009

Tuckerman, M., Berne, B. J., and Martyna, G. J. (1992). Reversible Multiple Time Scale Molecular Dynamics. J. Chem. Phys. 97, 1990-2001. doi:10.1063/1.463137

Van Holde, K. E., and Miller, K. I. (1995). "Hemocyanins," in Advances in Protein Chemistry (Elsevier), 1-81. doi:10.1016/s0065-3233(08)60545-8

Volbeda, A., and Hol, W. G. J. (1989). Crystal Structure of Hexameric Haemocyanin from Panulirus Interruptus Refined at $3.2 \AA$ Resolution. J. Mol. Biol. 209, 249-279. doi:10.1016/0022-2836(89)90276-3

Wang, J., Wolf, R. M., Caldwell, J. W., Kollman, P. A., and Case, D. A. (2004). Development and Testing of a General Amber Force Field. J. Comput. Chem. 25, 1157-1174. doi:10.1002/jcc.20035

Webb, B., and Sali, A. (2016). Comparative Protein Structure Modeling Using MODELLER. Curr. Protoc. Bioinformatics 54, 5.6.1-5.6.37. doi:10.1002/cpbi.3

Yoginath, S., Alam, M., Ramanathan, A., Bhowmik, D., Laanait, N., and Perumalla, K. S. (2019). "Towards Native Execution of Deep Learning on a LeadershipClass HPC System," in 2019 IEEE International Parallel and Distributed Processing Symposium Workshops (IPDPSW) (Rio de Janeiro, Brazil: IEEE), 941-950. doi:10.1109/IPDPSW.2019.00160

Zhong, T.-Y., Arancibia, S., Born, R., Tampe, R., Villar, J., Del Campo, M., et al. (2016). Hemocyanins Stimulate Innate Immunity by Inducing Different Temporal Patterns of Proinflammatory Cytokine Expression in Macrophages. J.I. 196, 4650-4662. doi:10.4049/jimmunol.1501156

Conflict of Interest: The authors declare that the research was conducted in the absence of any commercial or financial relationships that could be construed as a potential conflict of interest.

Publisher's Note: All claims expressed in this article are solely those of the authors and do not necessarily represent those of their affiliated organizations, or those of the publisher, the editors, and the reviewers. Any product that may be evaluated in this article, or claim that may be made by its manufacturer, is not guaranteed or endorsed by the publisher.

Copyright () 2021 Bux, Shen, Tariq, Yin, Moin, Bhowmik and Haider. This is an open-access article distributed under the terms of the Creative Commons Attribution License (CC BY). The use, distribution or reproduction in other forums is permitted, provided the original author(s) and the copyright owner(s) are credited and that the original publication in this journal is cited, in accordance with accepted academic practice. No use, distribution or reproduction is permitted which does not comply with these terms. 